\title{
XXXI.
}

\section{Die Geistesstörungen im Verlaufe des akuten Gelenkrheumatismus.}

\author{
Eine klinische Stndie. \\ Von \\ Dr. Th. Simon, \\ Ober-Arzt der 4. mediz. Abtheilnng des allgemeinen Krankenhauses zu Hamburg.
}

$\coprod_{\text {ie Geistesstörungen, welche im Verlaufe des akuten Gelenkrheuma- }}$ tismus auftreten, sind zwar schon in einzelnen Beispielen seit längerer Zeit bekannt, doch erst vor etwas über 12 Jahren durch Griesinger genauer gewürdigt. Im Jahre 1860 lenkte dieser vortreffliche Forscher durch seinen Aufsatz ,die protrahirte Form der rheumatischen HirnAffektion" die Aufmerksamkeit auf die in der Literatur zerstreuten Fälle, und seit dieser Arbeit ist die Casnistik von Zeit zu Zeit durch Mittheilung neuer Fälle und zusanmenfassende Besprechungen vermehrt. Zu letzteren rechne ich vor Allem die Arbeit Tüngel's ,über die nervösen Zufälle beim akuten Gelenkrheumatismus" in seinen ,klinischen Mittheilungen von der medizinischen Abtheilung des allgemeinen Krankenhauses zu Hamburg im Jahre 1860", ferner eine Berliner Inaugural-Dissertation von Julius Peyser aus dem Jahre 1867, welche gleich dem ursprünglichen Aufsatz Griesinger's die Bezeichnnng führt, ,über die'protrahirte Form der rheumatischen Hirn-Affektion" und 2 zusammengehörige Aufsätze von mir „über Geisteskrankheiten im Verlauf des akuten Gelenkrheumatismus" im 2. Heft des VIII. Bandes der Charité-Annalen (1865) p. 67-133 und der dazugehörige Nachtrag im XV. Bande (1869) p. 119-152. Die letzten beiden Arbeiten enthalten zugleich eine Besprechung und Zusammenstellung der früher beobachteten Fälle, nämlich: 4 bei Griesinger, 4 bei Bur- 
rows, 3-von Ulrich, 2 von Sander, 1 von Mugnier, 12 von Tüngel, 1 von Besser, 3 von Wille, 1 aus dem bayerischen Militairrapport, 1 von Ledru, 2 von Peyser und 18 Fälle von mir. Seit. der Zeit, in welcher mein letzter Nachtrag geschrieben, sind 2 nene Arbeiten über den uns hier beschäftigenden Gegenstand erschienen: von T. S. Clouston "Two cases of rheumatic insanity" (Journal of mental science. July 1870 vol. XV. p. 210) und die weit bedeutsamere von Rudolf H. Ferber in Hamburg, „Die nervösen Erscheinungen im Rheumatismus acutus" (Archir der Heilkunde Band X. 1869 p. 253) mit einer Fortsetzung „Rheumatismus, Chorea, Herz-Afektionen" (ibid. Bd. XII. 1871 p. 81).

Die Beobachtungen beider Autoren sind ausgezeichnet durch die Intensität der begleitenden motorischen Störungen, insbesondere der Chorea.

Clouston's I. Fall betrifft eine 27 jährige Matrosenfrau, die nach 2 Monate andauerndem subakuten Rheumatismus in den Gelenken der unteren und oberen Extremitäten 8 Tage vor der Aufnahme in die Irrenanstalt psychisch erkrankte. Als Ursache der Erkrankung tritt Anämie deutlich hervor unzureichende Ernährung, zu langes - 15 monatliches - Stillen neben Kummer und Unglück werden als ätiologische Momente aufgeführt, hereditäre Anlage fehlte. Erst stellte sich ein abnorm stilles Wesen, dann grosse Erregung ein, dabei heftige choreaartige Bewegungen der Arme, Beine und des Kopfes.

Bei der Aufnahme waren die Herztöne rein, Pupillen eng, Temperatur zwischen 37 und 38.

An Stelle der Chorea der Beine trat motorische und sensible Lähmung derselben. „Blase geläbmt, Patientin wurde 1 mal katheterisirt und kann seitdem den Urin lassen."

Die Genesung trat ailmählig nach einer Gesammtkrankheitsdauer von 4 Monaten ein.

Bei der Bedeutung, welche für die Diskussion der ,protrahirten rheumatischen Hirn-Affektion" die Recidive der Gelenk-Affektion haben - ein Thema, welches wir noch später berühren müssen - ist es wichtig, hervorzuheben, dass in diesem Fall zwei Recidive der GelenkAffektion beobachtet wurden, das erste im rechten Knie, wobei das Allgemeinbefinden sich verschlimmerte; das zweite nach 3 Monaten in dem rechten Handgelenk, das ohne Schmerzhaftigkeit anschwoll. Dies Recidiv war ohne Einfluss auf das psychische Befinden, von ungünstigem anf die Chorea.

Weniger rein ist der II. Fall Clouston's, da der Kranke, ein 19 jähriger Lehrling, exquisite hereditäre Anlage hatte: 
Der Vater war an Tetanus gestorben, die Mutter ,nervös", deren Schwester excentrisch, der Patient selbst schon früher der Chorea unterworfen. (2 Anfälle im 7. und 13. Jahre).

3 Wochen vor der Aufnahme erkrankte er an akutem Gelenkrheumatismus in den unteren und oberen Extremitäten, ohne Herz-Affektion. Kurz vor der Aufnahme trat Chorea in den Gliedern der linken Seite, Erregung, Verfolgungsideen und grosse Verwirrtheit ein.

Bei der Aufnahme erwies sich der Kranke als ein blasser, schmächtiger, hochaufgeschossener Mensch mit Chorea-artigen Bewegungen des Kopfes und der Glieder. Die Herztöne waren rein, die Handgelenke noch geschwollen. Die Temperatur bewegte sich eine Zeit lang um $38^{\circ}$, die höchste war $38,4^{\circ}$

Im Verlauf von 21/2 Monaten trat allmählig Besserung bis zur vollständigen Genesung ein.

Ferber's Arbeit, auf deren Bedeutung ich am Schluss meines letzten Aufsatzes schon hinweisen konnte, ${ }^{*}$ ) betrifft 2 Kinder.

Das erste hatte seit seinem 2. Lebensjahr alljährlich im Spätherbst Anfälle von akutem Gelenkrheumatismus gehabt. Bei einem im 8. Lebensjabre eingetretenen Recidiv, das sich besonders in den Hand- und Kniegelenken lokalisirte, trat Chorea der oberen, Schmerzhaftigkeit der unteren Extremitäten, Formikationen und Contractur derselben ein. Dabei bemerkte man ein scheues melancholisches Wesen ohne ausgesprochene Hallucinationen, ${ }^{* *}$ )

Ein im Marz 1869 eingetretener kurzer Anfall (in Handgelenken, Fingern, Knöcheln) verlief ohne psychische Störungen, im Spätherbst traten neue Anfälle auf, in denen das Kind starb. Die Untersuchung im Leben und die Sektion ergaben ausgedehnte Herz- und Lungen-Affektionen, Vergrösserung des Herzens, Verwachsung desselben mit dem Herzbeutel, starke Verfettung der Muskulatur, Mitralstenose; ferner ein frisches pleuritisches Exsudat auf der rechten, die Residuen einer überstandenen Pleuritis auf der linken Seite.

Weit schärfer tritt die psychische Veränderung bei dem anderen von Ferber beobachteten Kinde", einem $2^{2} / 3$ Jahr alten Knaben hervor. Das Kind hatte seipe Anlage zu nervösen Erkrankungen (der Grossvater ist epileptisch und gelähmt) schon durch eclamptische Anfälle bewiesen.

Im April 1869 erkrankte der Knabe an Rheumatismus der Sprunggelenke. Dabei zeigte sich tiefe Verdriesslichkeit und Abneigung gegen seine ihm sonst liebe Umgebung (Vater, Spielgefährten) nebst Chorea. Ein Recidiv im April 1870 (Fnssgelenke, Knie) brachte neben weniger ausgesprochener Chorea eine Herz-Affektion (Pericarditis) und wieder ein ganz auffäliges verdriessliches Wesen. Seit jener Zeit hat sich das Kind uach mir gewordener freundlicher Mittheilung meines Collegen Ferber bis jetzt (October 1873) gut gehalten und normal entwickelt.

Bei mancher Verschiedenheit haben die hier in kurzem Auszuge mitgetheilten Fälle Clouston's and Ferber's insofern grosse Aehn-

*) Charité-Annalen Bd. XV. p. 151; es ist dort irrthümlich "Gerber" gedruckt.

**) Aehnlich meiner IX. Beobachtung. Charité-Annalen XIII. 2. p. 89. 
Die Geistesstörungen im Verlaufe des akuten Gelenkrheumatismus. 653

lichkeit, als die Art der Geistesstörungen bei allen 4 eine mehr oder weniger reine Melancholie ist - nur bei Clouston's II. Fall eingeleitet durch eine kurze maniakalische Erregung - und alle 4 von eigenthümlichen motorischen Störungen, die mehr oder weniger dem Symptomencomplex der Chorea entsprechen, begleitet sind.

Um so wesentlicher erscheint es mir, dass hervorgehoben wird, wie dieses Bild durchaus nicht das gewöhnliche der rheumatischen Geistesstörung ist, und dass diese unter sehr verschiedenen Formen auftreten kann, wie die nachfolgenden Krankengeschichten beweisen werden. Die eine, im knrzen Auszug mitgetneilte, ist in den letzten Monaten in Frankreich veröffentlicht. Die anderen ausführlich beschriebenen, sind theils von mir auf meiner Abtheilung im allgemeinen Krankenhause und in der Privat-Praxis beobachtet, theils verdanke ich ihre Mittheilung der Freundlichkeit meines früheren Oberarztes, Herrn Dr. D. W. Reye, Oberarzt der Irrenanstalt Friedrichsberg, die vier letzten der Güte der Herren Direktor Dr. Rüppel nnd Dr. Hansen aus der Trrenanstalt bei Schleswig.

Ich habe zwar früher*) die Ansicht ausgesprochen, dass die Casuistik der Geistesstörungen nach akutem Gelenkrheumatismus abgeschlossen werden könnte, aber Angesichts der erwähnten Differenzen und der geringen Verbreitung, deren sich bis jetzt noch die Kenntoiss der „rheumatischen Psychosen" erfreut, scheint mir die ausführliche Mittheilung neuer Fälle gerechtfertigt.

J. Christian in Montdevergnes theilt in seiner Arbeit: de la folie consécutive aux maladies aigues, **) folgenden Fall ***) mit:

Ein 20jähriger bisher gesunder Fabrikarbeiter, erkrankte im Februar 1865 an akutem Gelenkrheumatismus. Von Anfang an ,bizarreries de langage", der Kranke entschuldigt sich beständig wegen der grossen Mühe, die er seiner Umgebung bereite.

Nach 3 Wochen mehrstündiger Anfall von Agitation, der vorübergeht, aber sich öfters wiederholt. Dazwischen Stupor und Verfolgungsideen; allmählig Besserung, so dass der Kranke Ende April wieder zur Arbeit gehen kann.

Aber schon am 14. Mai muss er sich wegen Rückfalls zu Bett legen: Fieber, Gelenkschmerzen ohne Anschwellung, ,Antworten langsam, schwierig (embarassées)". Am 20. Mai Tobsucht, so rasch zunehmend, dass Patient nach Stephansfeld transportirt werden musste. "Les douleurs rhumatismales paraissaient complètement disparues". Tod nach 14 Tagen in hoher Manie. Sektion negativ.

*) Charité-Annalen XV. p. 151.

**) Archives générales. September und 0ktober 1873.

**) 1. c. obs VI Septemberheft vol. XXII. p. 262. 
In diesem Fall ist also vom Anfang der akuten Krankheit an die Alienation zu erkennen, die Form derselben ist höchst wechselnd: Agitation, Melancholie, Manie.

Mit dem Recidir des Rheumatismus tritt ein Rückfall der Geistesstörung ein. Dass während der finalen Tobsucht von der Gelenkaffektion nichts bemerkt wurde, wird den nicht Wunder nehmen, der öfter Maniakalische trotz ihrer Verletzungen, Entzündungen u. s. w. herumtoben gesehen hat. In einer meiner eigenen Beobachtungen, die ich jetzt folgen lasse, werden wir einen Kranken herumspringen sehen, trotz der objektiv nachweisbaren Affektion der Fussgelenke. *)

\section{Erster Fall. (XXIY. Beobachtung) ${ }^{* *}$ )}

Aeusserst protrahirter Gelenkrheumatismus von fast 3 monatlicher Daver. Herzaffektion. Grosse Anämie. Frregungszustände, dann Melancholie und Stupor. Allmählige Besserung. Heilung nach ca. 4lmonatlicher Geistesstörung.

Heinrich Martin Wr., 22 jähriger Arbeiter aus Finkenwerder (einer der hamburgischen Elbinseln) wurde am 5. Febrnar 1868 in das allgemeine Krankenhaus aufgenommen. Er war vor 8 Tagen mit Schmerzen in den Phalangealgelenken der Finger erkrankt. Zugleich stellte sich Fieber und heftiger Schweiss ein. so dass Patient bettlägerig wurde. Später trat Anschwellung und Schmerzen in den Hand-, Knie- und Fussgelenken hinzu. Jetzt besteht dieselbe hauptsächlich in den Schultern. - Patient ist ziemlich wohlgenährt. Haut feucht. Zunge dick weiss belegt. An den obenbenannten Gelenken findet sich in verschiedener Intensität Schwellung und Röthung. Der Herzchoc ist verstärkt, der erste Mitralton nicht ganz rein, doch ist kein bestimmtes Geräusch wahrnehmbar. Stubl seit 2 Tagen ausgeblieben. [01. ricini; Anfangs mixt. nitros; 7. Februar, succus citri; 10. Februar, Kalium jodatum].

11. Februar. Die Gelenkschmerzen halten in mässigem Grade an: das Fieber ist etwas gesunken. Patient hat jetzt regelmåssig Stuhl.

19. Februar. Unter mässigem Fieber sind immer ein bis zwei Gelenke geschwollen und schmerzhaft. Die letzteren Beschwerden wechseln sehr rasch. Meist sind die Hand- und Kniegelenke befallen.

19. März. War einige Tage fast fieberlos und von allen Schmerzen frei. Seit dem 27. sind dieselben wieder in der rechten Schulter und dem rechten Handgelenk vorhanden. Auch das Fieber ist wieder etwas vermehrt.

15. April. Die Gelenkaffektion besteht in geringerem Grade noch in beiden Schultern. Der Ernährungszustand macht sehr langsame Fortschritte, obwohl Patient schon seit mehreren Wochen sehr guten Appetit zeigt.

24. April. Patient zeigt seit einiger Zeit ein eigentbümlich schen ver-

*) Dritter Fall (XXIV. Beobachtung).

**) I-XVIII im 2. Heft des XIII. Bandes der Charité-Annalen. XIX-XXI im XV. Band derselben. 
schlossenes Wesen. Zuweilen macht sich ohne äussere Veranlassung Unruhe und Aufregung bemerklich. Die Pulsfrequenz steigt dann plötzlich bis zu 112 und 120, um eben so schnell wieder abzufallen.

Heute Morgen war Patient aufgeregter als je, behauptete, in seinen Kleidern und im Bett Läuse $\mathrm{zu}$ bemerken, klagte dann darüber, dass er Trichinen habe etc. Er war nicht im Stande die gewöhnlichsten Handleistungen zu verrichten, benahm sich beim Bettmachen so ungeschickt, dass ihm der Wärter zu Hilfe kommen musste. Er hat bisher nichts genossen. Seine Antworten sind sehr spärlich, oft bleiben sie ganz ans. Er macht dann Bewegungen mit den Lippen, als ob er sich Mähe gebe zu sprechen. In diesem Zustande wurde er der Irrenanstalt äbergeben.

Hier wurde über ihn folgender Stat. praes. aufgenommen:

Er ist ein ziemlich lang aufgeschossener, schmal gebauter, junger Mensch, von auffallend blasser. Farbe und eingesunkenen Wangen.

Bei der körperlichen Untersuchung zeigen sich ausserdem abnorm:

die Zunge, die weiss belegt ist;

das Herz, dessen Töne undeutlich und gedehnt sind, zumal der erste Mitralton;

der Urin - der einen ziemlich bedeutenden Blasenkatarrh bekundet, übrigens kein Eiweiss enthält.

Patient blickt meist ängstlich und mit ernstem trüben Blick vor sich hin, spricht sehr wenig und meist nur nach wiederholten Fragen. "Sein Kopf soll von Läusen wimmeln, Trichinen in seinem Körper sein" etc.

Er geniesst fast nichts; gestern Mittag 2 Löffel Suppe - heute gar nichts. Nach der Ursache befragt, antwortet er scheu und leise: er dürfe ja nicht; er müsse für seine beiden Brüder mitleiden etc.

Der Schlaf mass sehr unzureichend sein, denn bäufig verlässt der Kranke Nachts und am Tage sein Bett, um sich alsbald wieder nieder zu legen.

29. April. Der Kranke hat heute Morgen sein Weissbrod gegessen, gestern Abend den Wein genommen; sagt fast nichts. Schläf wenig, antwortct fast gar nicht.

30. April. Aeusserst scheu und ängstlich; hält die gereichte Hand krampfhaft ängstlich fest. Blick starr. Spricht fast gar nicht, isst ron selbst nicht, sondern muss vom Wärter gefüttert werden; verlässt häufig das Bett, in unbestimmter Angst fortwollend.

3. Mai. Psychisch dasselbe Verhalten. Starker foetor ex ore; schwarzer Zungen- und Lippenbelag, Puls ziemlich kräftig, 50-60 in der Minute.

10. Mai. Isst freiwillig gar nicht, muss gefüttert werden; ist äusserst schwach und jämmerlich. - Spricht gar nicht, ist sehr scheu und ängstlich; hallucinirt stark.

15. Mai. Keine Veränderung. Dasselbe ängstlich widerstrebende Benehmen, sagte: er dürfe ja nicht essen. Ist auf's äusserste abgemagert.

25. Mai. Verlässt häufig sein Bett, legt sich in ein anderes, zerkaut stets seine Hemden, die Bettdecken, so dass ganze Stücke davon verschwinden. Mitunter isst er jetzt etwas besser. Beginnender Decubitus.

30. Mai. Der Decubitus breitet sich nicht weiter aus. Der Kranke fängt an, regelmässiger von selbst zu essen, spricht aber fast gar nichts, ausser ja und nein. 
10. Junj. Isst jetzt gut, Der Decubitus rerheilt, Der Gesichtsausdruck des Patienten ist etwas weniger ängstlich, er lächelt mitunter, spricht jedoch nicht.

26. Juni, Fängt an, sich zu erholen; isst gut; ist nicht mehr so widerstrebend; sitzt täglich ein paar Stunden im Lehnstuhl an der Luft. Zerkaut noch fortwährend seine Kleidung.

30. Juni. Wird kräftiger, kann schon allein gehen, wird voller. Spricht noch immer äusserst wenig und nur halblaut.

15. Juli. Fortschreitende Besserung und Kräftigung. Geht einen halben Tag mit zur Arbeit in's Freie. Ist jetzt freundlich, reicht die Hand, spricht jedoch nur noch wenig.

30. Juli. Wird voller und kräftiger, arbeitet fleissiger, ist oft fast heiter, lächelt. spricht etwas mehr und ganz vernünftig.

15. August. Wird körperlich immer voller und kräftiger, geistig frisch und munter, verkehrt mit den übrigen Kranken.

30. August. 4. Wochen Urlaub.

1868 5. October. Geheilt entlassen.

Im Sommer des nachfolgenden Jahres traf ihn einer der Aerzte auf einem Volksfeste; er sah kräftig und wohl aus und gab an, dass er rüstig bei der Arbeit sei. Ueber den Zustand des Herzens beim Abgange des Kranken findet sich leider nichts bemerkt. -

\section{Zweiter Fall. (XXII, Beobachtung).}

Akuter Gelenkrheumatismus mit Pleuritis links, Pericarditis und Endocarditis. Symptome psychischer Störung in der Zeit der Abnahme der GelenkAffektion; nach Heilung der Pleuritis ausgesprochene Melancholia agitata von fast 14 tägiger Dauer Pneumonie links mit Krisis am 3. Tage. Endocarditis. Wiederholte Recidive des Gelenkrheumatismus ohne psychische Störung. Nochmals linksseitige Pleuritis. Heilung mit zurückbleibender Insufficienz der Mitralis.

Meta Maria M. aus Freiburg a. d. Elbe, 29jähriges Dienstmädchen, wurde am 24. December 1872 auf meiner Abtheilung im allgemeinen Krankenhause aufgenommen.

Patientin, ein gut genährtes, blühend aussehendes Mädchen, gibt an, seit circa 8 Tagen krank zu sein: sie habe sich matt gefühlt, Schmerzen in den Füssen und Knieen gehabt und den Appetit verloren.

Bei der Anfnahme zeigt sich mässiges Fieber, die Fuss- und Kniegelenke sind geschwollen, geröthet, schmerzhaft, die Herzdämpfung beginnt an der 3. Rippe und reicht nach rechts bis an den rechten Sternalrand, nach links bis $1 \frac{1}{2} \mathrm{Cm}$. innerhalb der Mammillarlinie im $\nabla$. Intercostal-Raum, die Herztöne sind rein, der erste Ton etwas dumpf. Respirations-Organe vollständig normal.

Leber- und Milzdämpfung ist nicht vergrössert, die Zunge gelblich weiss belegt, feucht, Appctit fehlt, Stuhl retardirt. In dem mässig reichlichen Urin schlagen sich viel Urate nieder, Eiweiss ist nicht vorhanden.

Keine Kopfschmerzen, lebhafte Klagen über die intensiven Schmerzen, Schlaflosigkeit. [Natr, bicarb ]. 
27. December. Auf Morphium ist etwas Schlaf eingetreten, die Gelenke der unteren Extremitäten sind besser, dagegen das linke Elandgelenk sehr bedeutend geschwollen, dabei intensiv schmerzhaft. (Eisblase!)

Am Herzen hört man statt des ersten Tones ein deutliches systolisches Geräusch, die Dämpfung erscheint nach oben etwas verlängert, man hört deutliche Reibungsgeräusche, die Kranke klagt über Dyspnoe.

3, Januar. Anhaltend nur geringes Fieber. Die Herzdämpfung ist sehr verbreitert, reicht nach oben bis an die II. Rippe, nach rechts 2 Querfinger über den Sternal-Rand. Die Herztöne sind nur ganz schwach zu hören, dagegen sind sehr laute schabende und reibende Geräusche zu hören und zu fühlen. Puls klein, frequent (132), unregelmässig. Das rechte Handgelenk ist abgeschwollen, dagegen ist jetzt das linke schmerzhaft. [Inf. Digit. 1,0180,0. Eisblase auf das Herz].

8. Januar. Lebhaftere Dyspnoe, Klagen über Stiche in der linken Seite. Links unten hinten ist Dämpfung bis etwas über den angulus scapulae. Der halbmondförmige Raum ist vollständig ausgefüllt. Unten hört man gar kein Athmungsgeräusch, weiter oben an der Grenze der Därnpfung hauchendes Athmen. Die Gelenke sind wenig' schmerzhaft, auch die Handgelenke schwellen ab. Am Herzen ist die Dämpfung noch intensiver, die Geräusche sind verschwunden, man hört nur ganz entfernt undeutliche Töne. Puls 120. [contin. Digitalis.]

12. Januar. Das Fieber hat zngenommen, ist gestern bis $40^{\circ}$ gestiegen, der pleuritische Erguss ist bedeutend gestiegen. Die absolute Dämpfung geht bis zur spina scapulae, in ihrer ganzen Ausdehnung hört man gar kein Athmungs-Geräusch. Vorn scharfes bronchiales Athmen (comprimirte Lunge?) Die Gelenke sind jetzt frei. Auffällig ist das indifferente Verhalten der Patientin, die keine Dyspnoe klagt und etwas Appetit bekommt.

16. Januar. Das Fieber hat einen mehr intermittirenden Charakter, der Appetit hebt sich, die Gelenke sind frei, die Befunde am Thorax unverändert, die Kranke schläft fast den ganzen Tag, obschon sie kein Morphium bekommt; erweckt sagt sie, es ginge ganz gut. Dabei objective Dyspnoe (coupirte Sprache, Nasenflügel-Athmen).

22. Januar. Die Herzdämpfung ist ziemlich im Gleichen, man hört jetzt aber wieder Reibegeräusche. Die linke Pleurahöhle ist vollständig von Exsudat gefüllt, ausgedehnt, die Intercostalräume deutlich verstrichen. Nach Gebrauch von 5,5 Digit. wird dasselbe ausgesetzt, Patient erhält 500,0 aqu. crystallina pro die.

2. Februar. Im Zustand der Kranken ist eine bedeutende Besserung unverkennbar, das Plearaexsudat ist beträchtlich gesunken, steht handbreit unter der spina scapulae, die Lungenspitze giebt hellen Percussionston und resiculäre Athmung, die Hel'zdämpfung reicht bis zum obern Rande der III. Rippe, man hört ein systolisches Geräusch und einen zweiten Ton. Urinsekretion ist bedeutend vermehrt, der Stuhlgang etwas retardirt.

Patientin schläft noch immer auffällig viel, spricht im Schlaf allerlei vor sich hin, wird sie ganz wach, so giebt sie verständige Antworten.

5. Februar. Nach Angabe der anderen Kranken ,tütert" Patientin zuweilen (Provincialismus für: verwirrtes Zeug reden); bei der ärztlichen Visite 
ist dies noch nicht aufgefallen, vielmehr findet man sie fast immer schlafend. Das pleuritische Exsudat wird rasch resorbirt, die Iämpfung reicht aber noch bis zum angulus scapulae.

7. Februar. Die Kranke ist in der Nacht sehr unruhig gewesen, hat allerlei wirres Zeug gesprochen ,sie sei todt", "man wolle sie tödten" u. s. w. dabei versuchte sie, das Bett zu verlassen. [Ab. 2,0 Chloral.]

8. Februar. Trotz des Chloral nicht geschlafen; sie hält ihre Umgebung für todt, ,alle sehen todt aus". Ardere Wahnideen knüpfen an das Kindergeschrei an, welches sie aus der nebenliegenden Kinder-Krankenstube hören kann, ,sie habe guboren“, ,ihr Kind werde drinnen ermordet" u. s. w. Dabei fortschreitende Besserung aller objektiven Befunde.

10. Februar. Patientin hat trotz 3,0 Chloral nicht geschlafen, sie ist anch bei Tage sehr unruhig, schreit und stösst verwirrte Reden aus. Zwischendureh liegt sie stundenlang im Halbschlaf. Gegen $\Lambda$ bend war sie äusserst laut und unruhig, so dass sie isolirt werden musste. [3,5 Chloral.]

11. Februar. Hat gut geschlafen, ist heute aufiällig ruhiger, giebt aber keine Antworten, spricht leise vor sich bin, doch ist es nicht zu verstehen was sie sagt. [reit, 3,5 Chloral.]

12. Februar. Die Kranke hat das Chloral genommen aber die ganze Nacht nicht geschlafen, schrie so laut, dass die ganze Abtheilung gestört wurde. Auch bei Tage äussert sie beständig melancholische Wahnideen. Halbe Tage lang weigert sie sich, zu essen, Medizin einzunehmen, und ist ubberhaupt sehr widerstrebend.

Der Ernährungszustand ist dabei kein schlechter. Das Fieber hat vollständig aufgehört, die Gelenke sind frei, das pleuritische Exsudat ist bis anf geringe Reste vollständig resorbirt und man hört auf der Lunge überall vesiculäres Athmen mit einzelnen dumpfen Rasselgeräuschen. Der Urin ist reichlich, hellgelb, von niedrigem specifischen Gewicht und Eiweissfrei.

In den letzten Tagen liess die Kranke Urin und Stuhl öfter unter sich.

In diesem Zustand warde sie am 13. Februar 1873 zur Irrenanstalt Friedrichsberg gesandt.

Hier wurde folgender Status praesens aufgenommen: Patientin ist sehr abgemagert, hat am os sacrum eine Decubitusstelle, die im Verheilen begriffen ist. Schädelbildung nicht von der Norm abweichend. Pupillen etwas weit, Gesichtsausdruck ängstlich. Bei der Untersuchung des Thorax findet sich die Herzdämpfung vergrössert und zwar besonders nach oben und links. Die Herztöne sind rein. Hinten und links am Thorax hört man deutliches pleuritisches Reiben. Dabei reines Athmungsgeräusch und vereinzelte Rasselgeräusche, die auch an der Basis der rechten Lunge zu hören sind. Patientin hustet wenig, wirft nichts aus, klagt nicht über Schmerzen. In den Bauchorganen ist nichts Abnormes zu constatiren. Die Zunge ist ziemlich rein. Stuhlgang will sie täglich haben. Appetit mässig.

Psychisch zeigt sie sich bei ihrer Aufnahme sehr ängstlich, glaubt, man wolle ihr etwas anthun, ist sehr misstrauisch gegen das Wartpersonal, wird verdriesslich, wenn man sie etwas fragt.

18. Februar. Da Patientin gestern Abend um 11 Uhr noch nicht eingeschlafen war, bekam sie 2,0 Chloral, worauf sie bis hente Morgen rulig schlief. Gibt an, dass sie allerlei Gestalten, die ihr unbekannt waren, an ihrem Bett- 
ende gesehen habe. Nachmittags will sie verschicdene sehr schöne Farben gesehen haben; bei der Visite deutet sie noch auf eine bunte Fahne, die an der Wand hängen soll. Bekommt kräftige Diät and ein Infus. rad. Ipecac. c. Morph. Wasserkissen.

19. Februar. Die Kranke hat heute Nacht ohne Arzneimittel geschlafen: sie giebt etwas langsame Antworten, die jedoch immer richtig sind, ihr Allgemeinbefinden ist erträglich, der Gesichtsausdruck ist etwas heiterer. Gestern Abend hat sie schwarze Männer gesehen. Der Befund auf den Lungen unverändert.

21. Februar. Will nicht mehr an Gesichtstäuschungen leiden, lacht, wenn man sie darnach fragt. Sie liegt ruhig za Bett und soll sich mit der Wärterin ganz verständig unterhalten. Schlaf und Appetit gut. Temperatur Morgens und Abends normal. Puls 90, ziemlich klein. Zahl der Respirationen 20 in der Minute.

23. Februar. Der Decubitus ist beinah verheilt. Die Patientin hat etwas mehr Hustenreiz, die Herzaction ist noch sehr stürmisch, die Herztöne sind jedoch rein.

25. Februar. Die Rasselgeräusche sind an der rechten Lunge geschwunden, links noch hörbar, das pleuritische Reiben hat ebenfalls abgenommen. Herzdämpfung noch nicht verkleinert.

27. Februar. Unveränderter Zustand. Die Kranke äussert keine Wahnideen. Hält sich sehr ruhig.

28. Februar. Erkrankte heute Nachmittag plötzlich mit einem Frostanfall, heftigen Schmerzen in der linken Seite und mehrmaligem Erbrechen. Temperatur am Abend 40,1. Puls 132. Starke Dyspnoe. Hustenreiz 'mässig.

1. März. An der linken Lunge leerer Schall bis zur Mitte der Scapula; deutlich bronchiales Athmen, Stimmfremitus verstärkt. Die Dyspnoe ist hochgradig. Blutige Sputa werden nicht ausgeworfen. Die Temperatur schwankt zwischen 39,5 und 40,3 , Puls 128 .

An der Herzspitze hört man statt des ersten Tons ein leichtes blasendes Geräusch. Patientin hat nichts gegessen, ihre Zunge ist mässig belegt. [Verordnung: Tinct. Jod. äusserlich. Flor. Benz. 3 stündlich. Portwein.]

2. März. Der Befund an der Lunge ist derselbe, das Allgemeinbefinden aber besser. Die Kranke hat wenig geschlafen, sie wirft fast gar nichts aus. Temperatur Morgens 39,1, Abends 40,2. Puls 124, Abends 132.

3. März. Ist heute Morgen fieberfrei. Kann rubig Luft holen. Statt des Bronchialatbmens hört man knisternde Rasselgeräusche. Bis zur Spitze der Scapula noch leerer Schall (Exsudat). Stimmfremitus vermindert. [Infus. rad. Ipecac.]

5. März. Herzdämpfung gross. Geräusch noch vorhanden. Zweiter Pulmonalton accentuirt. Schläft ruhig. Psychische Störungen treten nicht zu Tage.

8. März. Die Dämpfung ist fast ganz verschwunden. Feuchte Rasselgeräusche im linken Unterlappen. Patiention ist fieberfrei gebliebeu. Hat guten Appetit.

12. März. Patientin klagt über nichts. Hat sich sichtlich erholt. Schlaf sehr gut.

16. März. Ist ruhig und verständig. 
20. März. Steht Nachmittags auf, soll sich aber sehr ruhig halten, da sie leicht Herzklopfen bekommt.

26. März. Sie weinte, ohne dass sie einen Grund angab weshalb.

29. März. Hatte heute Stiche in der Herzgegend und leichte Temperaturerhöhung. Bleibt liegen. Die linke Brust wird mit Tinct. Jod. bepinselt.

30. März. Schmerzen verschwunden. Puls ruhig. Kein Fieber. Das blasende Geräusch an der Herzspitze besteht unverändert fort.

2. April. Ist fieberfrei geblieben. Kein Herzklopfen. Schläft rahig.

6. April. Ist aufgestanden und beschäftigt sich wieder.

11. April. Nimmt seit einiger Zeit Tinčt. Ferr. pomat. Verträgt dieselbe gut.

16. April. Arbeitet sehr fleissig. Keine Symptome von psychischer Depression.

30. April. Ist sehr nett und ordentlich. Hat bedeutend an Körpergewicht zugenommen. Rasselgeräusche sind in der Lunge nicht zu hören.

6. Mai. Klagt über Schmerzen in den Knie- und Schultergelenken, die aber nicht sehr heftig sein sollen. Hat zeitweise wieder Herzklopfen. Herzdämpfung in beiden Durchmessern bedeutend vergrössert. An der Herzspitze leichtes systolisches Geräusch.

10. Mai. Hat jetzt Schmerzen in den verschiedensten Gelenken, die von einem nach dem andern ziehen, bald intensiver, bald schwächer sind. Keine Anschwellung. Allgemeinbefinden nicht beeinträchtigt. Kein Fieber. Urin ohne Eiweiss. [Bekommt Bäder aus Kal. sulph. pro balneo und innerlich Natr. bicarb. Crem. Tart. Lith. carb. und Sach. ana. 3 mal täglich eine Messerspitze voll.]

12. Mai. Fast sämmtliche Fingcrgelenke sind angeschwollen und sehr schmerzhaft. Dieselben werden mit Tinct. Jod. bepinselt und in Watte gehüllt.

15. Mai. Die Schmerzhaftigkeit hat nachgelassen.

20. Mai. Hat in beiden Schultergelenken nur noch wenig Schmerzen.

22. Mai. Steht einige Stunden auf.

30. Mai. Schulter- und Ellenbogengelenke wieder schmerzhafter. Bleibt liegen. Psychisch normal.

3. Juni. Wird nach dem allgemeinen Krankenhause zurückverlegt.

Hier klagt sie jetzt noch über Ziehen und Steifigkeit der Gelenke, dieselben sind indess nirgends geschwollen oder auf Druck empfindich. Sie ist wohl, sieht gut aus. Die Percussion und Auscultation der Lunge ergiebt normale Verhältnisse. Die Herzdämpfung beginnt am 3. Intercostalraum, reicht medianwärts bis zum linken Sternalrand. Der Herzstoss liegt nach aussen und unten ron der Brustwarze. Man hört ein der.tliches systolisches Gerïusch, der 2. Ton ist etwas verstarkt. Der Appetit gut. Stuhlgang regelmässig, psychisch ist die Kranke vollständig normal. [Kalium jodatum.]

4. Juni. Sie klagt über Schmerzen und Druckempfindlichkeit in beiden Schultergelenken. [Zu Bett.]

7. Juni. Die Schmerzen sind geschwunden. Patientin steht auf.

8. Juni. Schmerzen und etwas Druckempfindlichkeit in beiden Knieen, bleibt im Bett.

17. Juni. Steht wieder auf. Gelenke frei.

13. Juli. Seit dem 10. Juli Fieber. Patientin klagt über Stiche in der 
linken Seite und Kurzluftigkeit, Hinten unten links bis zum angulus scapulae unregelmässig mit der hinteren Axillarlinie abschneidend, Dämpfung mit aufgehobenem Athmen, Appetit gering, Zunge belegt.

18. Juli. Seit dem 15. fieberfrei, das pleuritische Exsudat links ist im Abnehmen und war am 26. Juli geschwunden, die Patientin befand sich wieder ganz wohl und konnte am 12. October geheilt entlassen werden.

Die Verbreiterung der Herzdämpfung und das systolische Geräusch, so wie die Verstärkung des 2. Pulmonaltons bestanden noch bei der Entlassung. (Insufficienz der Mitralklappe.)

\section{Temperatur - Messungen.}

Die Messungen wurden 2 stündlich gemacht In der nachfolgenden Tabelle sind die am 9 Uhr Morgens und 9 Uhr Abends gefundenen Werthe angegeben, die in Klammern bcigefügten Zahlen bezeichnen die höchste und niedrigste, überhaupt an dem betreffenden Tage beobachtete Temperatur, falls diese nicht in die 9. Stunde gefallen.




Es ist eine lange Leidensgeschichte, auf die wir hier zurückblicken, eine Aufeinanderfolge von Krankheiten, die beinahe einen jährigen Zeitraum ausfüllen, von dem die Geistesstörung nur einen geringen Theil einnimmt. Charakteristisch zeichnet sich der Beginn der Alienation ab durch die subjective Indifferenz gegen die objectiv nachweisbare Verschlimmerung aller Symptome. Wir werden den Fall bei der resumirenden Diskussion noch zu verwerthen haben und wollen hier nur auf die Differenzen in der Art der Geistesstörung von der in der vorhergehenden Beobaçhtung hinweisen. Dort eine Melancholia cum stupore, hier eine Melancholia agitata, dort Stumpfheit, Verfolgungswahnideen, hier lebhafteste Erregung, lantes Geschrei, glänzende Bilder, dort eine mehrmonatliche, hier eine ganz kurze Dauer. Der nachfolgende Fall zeigt wieder ganz andere psychische Symptome und darf wohl anch als Beispiel einer im. Privathause mit absolutem No-restraint durchgeführten Tobsuchtsbehandlnng gerade das Interesse der praktischen Aerzte beanspruchen.

\section{Dritter Fall. (XXIV. Beobachtung).}

Vorausgegangene längere geistige Anstrengungen bei hereditärer Anlage zu Nervenkrankheiten. Akuter Gelenkrheumatismus von grosser Intensität, frühzeitig mit psychischer Störung. Peri- und Endocarditis. Schärferes Hervortreten der Geistesstörung mit Nachlass des Fiebers, trotz fortbestehender Gelenkaffektion. Manie mit melancholisch gefärbten Intervallen. Recidiv der Gelenkaffektion, ohne Besserung der Geistesstörung. Allmählige Besserung bei Fortbestehen einzelner Wahnideen, Charakteränderung, Albernheit. Genesung nach ca. 4 monatlicher Krankheitsdauer mit zurückbleibender Insufflclenz der Mitralis, ohne subjective Beschwerden.

August R., der "20 jährige Sohn eines Gutsbesitzers in der Nähe von Hamburg, stammt aus einer Familie, in welcher Nervenkrankheiten nicht selten sind. Seine beiden Grossmütter haben an Dementia senilis gelitten, welche bei der väterlicherseits einen sehr hohen Grad erreichte. Der Vater (und zeitweilig auch eine Schwester) leidet seit vielen Jahren an Blepharospasmus, die Mutter ist in jugendlichem Alter ganz plötzlich gestorben. A. R. selbst war als Kind inmer sehr zart und blass gewesen, hat viel an Kopfschmerzen und Nasenbluten gelitten. In den letzten Jahren, besonders seit er die Schule verlassen, hatte er sich bedeutend herausgemacht, war stärker und kräftiger geworden, wozu das freie Leben auf dem Lande viel beigetragen. Im Sommer 1872 musste er sich auf das Freiwilligen-Examen vorbereiten, viel arbeiten und still sitzen, was ihn besonders während der heissen Tage sehr angriff:*)

*) Ich führe dies an, weil die starke geistige Anstrengụng zum Examen entschieden zu Hirnleiden disponirt. Zweimal sah ich den Ausbruch einer tuberculösen Meningitis unmittelbar an die Arbeiten zum Freiwilligen-Examen anknüpfen. 
In den ersten Tagen des Oktobers hatte er eine leichte Angina mit Magenkatarrh, die ihn einige Tage an das Bett fesselten. Kaum genesen machte er einen Ball mit. Gleich danach fühlte er sich unwohl, reiste aber noch am 22. auf der Bahn einige Meilen. Am 23. musste er das Bett hüten, hatte wenig Fieber $\left(38^{\circ}\right)$, leichte Schmelzempfindung in allen Gelenken, starken Schmerz und bedeutende Anschwellung im rechten Sprung- und im Metacarpalgelenk der rechten grossen Zehe.

Die Herzdämpfung war nicht verbreitert, reichte nach oben zur 4. Rippe, nach rechts an den linken Sternalrand. Höhe und Breite $91 / 2 \mathrm{Cm}$. Spitzenstoss in der Mammillarlinie des 5. Intercostalraums. Die Töne rein.

24. Oktober. Patient hat fast gar nicht geschlafen, die Schmerzen im rechten Fuss sind mässiger, dagegen im rechten Knie bedeutend heftiger, einige Stunden später aueh intensive Schmerzen im linken Knie. Rückenschmerzen, nach dem Abdomen ausstrahlend; hochgradige Flatulenz.

Die nächste Nacht war sehr unruhig, der Kranke phantasirt, das Fieber nahm zu. Der rechte Fuss und die Kniegelenke sind sehr stark geschwellt und bei leisester Berührung äusserst schmerzhaft, Patient ist psychisch tief deprimirt; ,er sei so gnatterig", „möchte Alleszerhauen“, nichts ist ihm recht, seine Umgebung wird durch ihn auf das äusserste gequält. Nachmittags verfällt er ganz plötzlich in Schlaf, aus dem er delirirend gegen $7 \mathrm{Uhr}$ Abends erwacht. Die Delirien dauern die Nacht über an, am folgenden Tage war das Sensorium frei, dagegen zeigte sich jetzt Verbreiterung der Herzdämpfung (auf $12 \mathrm{Cm}$.) nach rechts (bis zur Mitte des Sternum) und oben (3. Rippe), der erste Ton war diffus.

Während die Schwellung der Sprung- und Zehengelenke nachliess, die der Kniee andauerte, wurden in den nächsten Tagen rasch nach einander fast alle grösseren und kleineren Gelenke der oberen Extremitäten von Anschwellung und Schmerzhaftigkeit befallen, besonders intensiv die Schultern. Der Kranke musste gefüttert werden, da er die Hände nicht bewegen konnte, der Appetit war sehr gering, die Lippen trocken, rissig, die Zunge dick belegt, dabei konstant hohes Fieber.

So bielt die Erkrankung in den nächsten Tagen an, während die Herzdämpfung nach rechts und oben sich noch weiter ausdehnte und ein exquisites systolisches Geräusch auftrat.

Gleichzeitig erwies sich aber der Kranke psychisch verändert: während er gegen seine Umgebung im Ganzen freundlichor wurde, zeigte er plötzlich eine intensive Abneigung gegen eine nahe Verwandte, die er unter rohen Schimpfworten aus dem Zimmer jagte.

In der ersten Novemberwoche nahmen das Fieber und die Gelenkschmerzen $a b$, der Kranke war sehr abgemagert, die Oberschenkel ödematös. Im Urin kein Eiweiss. Am Herzen lautes systolisches Blasen. Als Patient auf ein Wasserkissen gelegt werden sollte, sträubte er sich anfangs sehr heftig dagegen, „das ginge nicht an, er könne das nicht verlangen, es sei zu theuer, sein Vater konne das nicht bezahlen".

Am 9. November war der Geburtstag seines Bruders. Er hatte dazn grosse Einkäufe machen lassen, während er sonst sehr, sparsam wax. Statt diese Geschenke dem Bruder zu überreichen, giebt er ibm einige alte Sachen. Zur Rede gestellt, erklärt er, ,er habe den Bruder prüfen wollen" und über- 
reicht demselben mit einer ganz übertrieben feierlichen Rede die wahren Geschenke.

Am Nachmittag wird ihm ein Glas Wein gebracht, während er dasselbe hält und dabei spricht, starrt er plötzlich in die Luft, und mit erhobenem Arm das Glas haltend, blickt er minutenlang nach demselben Punkt, worauf er, als sei nichts geschehen, im Gespräch fortfährt und seinen Wein austrinkt.

Das Fieber war fast geschwunden (wenige Zehntel über $38 \%$. Die Gelenke kaum geschwollen, nur noch auf Druck empindlich, das pericardiale Exsudat nnd das systolische Geräusch bestanden fort, der Puls war.klein, frequent.

Der Kranke, der den ganzen folgenden Tag (10. November) in eigenthümlich gehobener Stimmung gewesen, liess gegen Abend die ganze Familie an sein Bett rufen ,er wolle sie noch einmal sehen, um Abschied zu nehmen, da er sterben müsse"،

Jetzt wurden alle aus seiner Twgebung entfernt, ein Krankenwärter besorgt und der Kranke beruhigte sich zunachst.

Aber nicht auf lange Zeit. Schon der nächste Morgen zeigte den Kranken zwar fieberfrei, aber erregt, unruhig, bald ängstlich, bald abnorm heiter. Die Gelenke waren geschwollen, mässig schmerzhaft, die Herzdämpfung sehr verbreitert, reicht nach oben bis zur 2. Rippe, nach rechts mehrere Querfinger breit über den rechten Sternalrand hinaus. Dabei hörte man ein systolisches Geräusch und schwachen 2. Ton. Der Pals war dauernd klein, frequent, unregelmässig Milz nicht vergrössert. Die Geistesstörung, die sich jetzt als das hervorragendste Symptom zeigte, war eine höcbst eigenthümliche. Einige Stunden lang war der Kranke tief melancholisch, weinte und schrie ,alle seien todt, durch seine Schuld, er sei ein Sünder, ein Beest", dann wieder lag er ganz steif und starr, „er sei eine Leiche“, will nichts essen, „da er todt sei“. Dann wieder will er mit Gewalt das Bett verlassen und trotz der hochgeschwollenen und gerötheten Fussgelenke und Knie springt er auf, lässt sich aber vom Wärter leicht zurückbringen. Zuweilen lockt er halbe Stunden lang seinen Hund, der gar nicht zugegen, dann bellt er selbst Stunden hindurch wie ein Hund und nimmt das Essen schnappend gleich einem solchen.

Der Appetit war wechselnd, bald ass der Kranke gierig alles ihm gereichte Essen, bald stiess er es von sich Der Leib war aufgetrieben, der Stuhl retardirt, liess sich durch Clysmata oder Electuarium c. senna aber hervorrufen.

Die Behandlung bestand in der Anwendung starker Morphium-Injectionen $(1 / 2$, schliesslich $3 / 4$ Spritze der gewöhnlichen $5 \%$ Morphiumlösung, nach Umständen 2-3 ständlich wiederholt) neben Tr. Digitalis und Eisblase auf dem Herzen, da der Kranke bier unangenehme Sensationen hatte, die er natürlich sofort in Wahnideen umsetzte: ,or habe eine Schlange auf dem Herzen, die gefüttert werden müsste".

Vor die Fenster wurde ein mit Drahtgeflecht besetzter Holzrahmen in die Mauer eingeschoben, und 2 Wärter waren immer in der Krankenstube, da der Kranke sich dann leichter fügte und keine unnöthigen Balgereien vorkamen.

In den ersten Tagen des December trat ein Recidiv der Gelenkaffektion, 
besonders in Zehen, Knieen und mehreren Fingergelenken ein, während die Temperatur nur um wenige Zehntel über $38^{\circ}$ stieg und bald zur Norm zurücksank. Am ersten Tage glaubte ich, dass die Geistesstörung sich gebessert, da der Kranke Iuhiger wurde, allein bald zeigte sich, dass dieselbe nur die Form gewechselt; an die Stelle der Manie war eine stupide Melıncholie getreten, der Kranke lig so ruhig, well er sich für todt hielt, die Anwendung prolongirter warmer Bäder und halbgräniger Opiumdosen erwieseu sich gegen die heftigen Gelenkschmerzen sehr wirksam, der Kranke schlief besser, doch musste er noch immer sehr sorgsam überwacht werden, da er plötzlich aus der scheinbaren Ruhe zu Tobsuchtsanfällen überging, schlug und biss. Zwischendurch plauderte er gleich einem 6jährigen Kinde, stotterte auch öfter, doch machte dies mehr den Eindruck des Absichtlichen. Das pericarditische Exsudat resorbirte sich in dieser Zeit bedeutend. Mitte December war die Gelenkaffektion bcseitigt, gleichzeitig der Kranke auch psychisch bedeutend ruhiger. Es trat jetzt öfter Nasenbluten auf, das durch Eisumschlägo gestillt wurde. Der Appetit des Patienten wurde jetzt ein ungeheurer, er ass wirklich unglaubliche Quantitaten, besonders Fleisch, das nebst grossen Massen besten Rothweins die Hauptnahrung des skelettartig ahgemagerten Kranken ausmachte., Ich liess ihn in dieser Zeit seinen Vater einen Augenblick sehen, er freute sich sehr mit ihm ,da er gemeint habe, er sei todt".

Bei Gelegenheit einiger kleiner, dem Kranken gemachten Weihnachtsgeschenke äusserte er wieder melaucholische Ideen, „das sei sehr schön, aber nicht für ihn, er sei die schönen Sachen nicht werth" u. s. w.

Nenjahr 1873 hatte sich der Kranke bedeutend gebessert, sah viel wohler aus, begann an Gewicht zuzunehmen. Meist lag er still zu Bett, doch sass er zuweilen im Lehnstuhl auf, bei den kürzeren mit ihm geführten Gesprächen merkte man wenig von ysychischer Störung, seinem Wärter gegenüber äusserte er aber öfter Sélbstmordideen. Iu dieser Zeit wurde das Drahtoitter entfernt, der Kranke hatte auch seit Mitte December nur einen Wärter bei sich.

Mitte Januar ging er bei schönem Wetter öfter an die Luft, er war scheinbar psychisch frei, aber er hatte ein eigenthümlich pedantisches, geziertes Wesen, das ihm sonst fremd gewcsen. Er sprach die unbedeutendsten Sachen mit feierlicher Stimme, war knauserig und schalt über kleine, für ihn gemachte Ausgaben.

Mitte Februar sandte jch A. R. auf die Besitzung eines Freundes seines Vaters. Als ich ihn Ende dis Monats aufsuchte, war er kaum wieder zu erkennen, so stark war er geworden. Er klagte jetzt über öftere Kopfschmerzen, Nasenbluten und Herzklopfen, das systolische Geräusch war sehr laut und blasend. Beim Treppensteigen schmerzten die Fussgelenke noch immer. Der Gutshelr, welcher den Kranken von früher her genau kannte, fand ihn noch albern, zuweilen redete er noch geradezu Unsinn. Auch diese letzten Spuren schwanden im Monat März, R. beschäftigte sich auf dem Gute, ging auf die Jagd, hatte vorzüglichen Appetit und wurde zunehmend dicker. Im Mai trat A. R. als Volontair in ein Hamburger Geschäft ein, in welchem er sich bald das vollste Zutrauen seiner Principale erwarb. Subjektiv hat er bis heute (December 1873) ron seiner Herzhypertrophie mit Insufficienz der Mitralis durchaus keine Beschwerden. 
Das Interesse dieses Falles liegt darin, dass er in der PrivatPraxis beobachtet wurde - neben den Ferber'schen wohl der einzige Fall in der Casuistik der „Rheumatismus-Psychosen“.*) So war eine genaue Kenntniss des Kranken vor dem Rheumatismus, so eine sorgsame Beachtung desselben nach der Krankheit möglich und liess zu einer Zeit, wo für gewöhnliche Hospitalbeobachtong der normale Geisteszustand zurückgekehrt schien, an der Aendernng des Charakters, an scheinbar ganz kleinen Zügen, das Fortbestehen eines krankhaften Zustandes erkenuen. Im Hospital hätte ich die Krankheit anf 2 Monate berechuet, in der Privat-Praxis konnte die doppelte Dauer konstatirt werden.

Von grossem Interesse ist das Verhalten des Kranken zu der Gelenkaffektion: Anfangs die heftigsten Schmerzen, während er nachher herumtobte, obschon die intensive Schwellung das Fortbestehen der Gelenkaffektion bewies; ferner die absolute Einflusslosigkeit des Rheumatismus-Recidiv auf die Besserung der Geistesstörung. Diese änderte sich nur in der Form. Die Geistesstörung schliesst sich endlich an die Delirien anf der Höhe der Krankheit an (gleich dem Christian'schen Fall) und von vornherein, schon wäbrend des Fiebers zeigen sich eigenartige Züge, wie sie den gewöhnlichen Fieber-Delirien nicht eigen sind.

Wieder ein anderes Verhalten finden wir im:

\section{Vierten Fall, (XXV. Beobachtung).}

Akuter Gelenkrheumatismus eines Individuum mit Schrumpfniere. Pericarditis, Pleuritis. Tiefer Stupor bei bestehender Affektion mehrerer Gelenke. Pneumonie. Zoster costalis. Zoster costo-abdominalis. Scheinbar tiefe Dementia, allmählige Besserung bei Fintritt eines Reoidiv im Sprunggelenk. Heilung. Dauer der Geistesstörung ca. 7 Monat. Tod unter Frscheinungę der urämischen Intoxication. Autopsie: Schrumpfniere, Verwachsung des Herzens mit dem Herzbeutel, Herzhypertrophie ohne Klappenfehler: Pia cerebri getrübt und verdickt.

Gottfried Jürgen P. aus Norburg, 34 Jahr alter Arbeitsmann, wurde den 28. November 1871 auf der IV. medizinischen Abtheilung aufgenommen.

*) Die Beobachtung von Geistesstörung nach akutem Gelenkrheumatismus muss in der Privat-Praxis zu den grössten Seltenheiten gehören. Eine Anfrage bei mehreren meiner beschäftigtesten Collegen ergab von allen eine verneinende Antwort. Erst nach Abschluss dieser Arbeit erhalite ich durch freundliche Mittheilung des Herrn Dr. Eschenburg, Arztes der Irrenanstalt in Lübeck, Kenntniss von einem wohl hierher gehörigen Fall. "Ich erinnere mich nur ein es Falles aus meiner Privat-Praxis, in dem Gelenkrheumatismus und Fieber plötzlich aufhörten und statt ihrer Delirien eintraten und als diese nach circa 14 Tagen verschwanden, der Rheumatismus mit geringem Fieber wieder zurückkehrte." 
Derselbe ist vor 10 Tagen mit Fieber, Kopf- und Brustschmerzen, 'Anschwellung der Gelenke, namentlich der Kniee, erkrankt und seitdem bettlägerig gewesen. Patient zeigt ein leicht icterisches Colorit. Die Herzdämpfung ist nach allen Richtungen vergrössert, ein Dreieck mit nach oben gerichteter Spitze bildend. Herztöne rein, aber nur sehr schwach neben einem mässig intensiven Reibegeräusch hörbar. Puls 84, kräftig. In beiden Kniegelenken mässiges Exsudat, active und passive Bewegungen erschwert und schmerzhaft. Expectoration spärlich.

2. December. Patient klagt über stärkeren Hustenreiz und grosse Dyspnoe. Links hinten unten findet sich an der Grenze des oberen und unteren Lungenlappens eine 3 fingerbreite Dämpfung mit lautem Compressionsathmen.

5. December. Pleurit. Exsudat geht zuräck. Dyspnoe geringer.

9. December. In beiden Kniegelenken noch beträchtliche Exsudate. Pericardiale Dämpfung und Reibungsgeräusche haben bedeutend abgenommen. In diesem Zustand ging Patient am 13. December ungeheilt ab, wurde jedoch schon am 19. December wieder auf die Abtheilung gebracht.

Er war jetzt in völlig "bewusstlosem" Zustande, die Herzdämpfung be= deutend vergrössert, von der 3. Rippe beginnend, seitlich den rechten Sternalrand überragend. Die Herztöne und Puls schwach; hie und da hörte man etwas schwaches, pericardiales Reiben, während die Herztöne an und für sich rein erschienen. Links die Symptome einer pneumonischen Infiltration (Dämpfung and Bronchialathmen) besonders hinten und seitlich. Beide Schultergelenke und Handgelenke sind geschwollen und anscheinend schmerzhaft. Patient spricht beständig vor sich hin, schreit öfter laut auf. Nachts ist er sehr unruhig gewesen. Der Urin ist ziemlich stark eiweisshaltig, dunkelbraunroth. Patient lässt Stuhl und Urin unter sich. (2 stündlich 1 Esslöfel Portwein).

\section{Temperaturen.}

19. December. 20. December. 21. December. 22. December. 23. December. Morg. Abds. Morg. Abds. Morg, Abds. Morg, Abds. Morg, Abds.

$\begin{array}{lllllllll}39 & 37,4 & 38 & 38,2 & 38,4 & 37,6 & 38,2 & 37,4 & 37,2 \\ 39,4 & 38 & 38,4 & 38,4 & 38,8 & 38 & 37,6 & 38 & 37,6 \\ & 38,6 & 38,6 & 38 & 38,4 & 37,6 & 37,2 & 37,6 & 37,2 \\ & 39 & 39,2 & 38,6 & 39 & 37 & 37,6 & 37,6 & 37,6\end{array}$

26. December. Patient in den ersten Tagen sehr unruhig, hat ofter aufgeschrieen, ist Nachts aufgestanden und hat beständig irre geredet, liegt jetzt ruhig und apathisch da, reagirt nur sehr träge und ist jetzt völlig alienirt, erkannte seine eigene Frau nicht. - Das Fieber ist abgefallen, der Puls kräftiger, seit einigen Tagen źeigte sich herpes labialis. Da der Kranke durch seine Unruhe und Irrereden äusserst störend war, musste er am 1. Januar 1872 zur Irrenstation verlegt werden.

Auch hier beantwortete er keine Fragen, lag meist still, war jede Nacht schmutzig. Dabei sah er blass und abgemagert aus. (Warmes Bad täglich).

13. Januar. Er hat an der rechten Thoraxhälfte ganz herumziehend eine in parallelen horizontalen Reihen geordncte, in der Lebergegend sich ca. $7 \mathrm{Cm}$. in die Höhe erstreckende Herpes-Eruption, die vorn noch im Bläschen, hinten 
im Borkenstadium ist. Jede Berührung der Seite ist ihm äusserst schmerzhaft. Der ganze Herpesgürtel liegt in der horizontalen Ebene, ist also nicht den Nv. intercostales, sondern vorn den Nv. cutan. lateral. ant. und post., hinten den Rami post. der Dorsalnerven entsprechend.

29. Januar. Herpes überall eintrocknend und vernarbend.

2. Februar. Nachdem Patient die letzten Nächte rein gewesen war, ist er heute Morgen wieder nass.

29, Februar. Ist heute auf und befindet sich ganz wobl dabei. Steht freilich meist an einem Flecke.

3. März. Sein Gang ist sehr langsam und unsicher. Er antwortet mitunter ja oder nein, weitere Auskunft ist von ihm nicht zu erhalten. Nachts ist er häufig nass, aber jetzt viel seltener schmutzig als im Anfang.

12. April. Wird ungeheilt in die Irrenanstalt seiner Heimath, Schleswig, abgeholt. Auch in Schleswig stand er anfangs gesenkten Hauptes an einem Fleck, alle an ihn gerichteten Fragen höchstens mit "ja a und "nein " beantwortend, von selbst spricht er gar nichts. Nachts lässt er Urin unter sich, hält sich von den übrigen Kranken abgesondert.

Der Prls ist verlangsamt, die Hände bläulich und kalt, die Füsse geschwollen. Der Schlaf ist ruhig, Appetit gering. - Körperlänge $167 \mathrm{Cm}$., Gewicht 110 Pfd. (Warme Bäder, roborirende Diät, acid. phosph.)

26. April. Unverändert, spricht gar nicht, hat sich in der letzten Zeit nicht wieder nass gemacht. Urin frei von Eiweiss, enthält phosphorsauren Kalk.

4. Mai. Wird etwas reger, spricht pon selbst, klagt über Schmerzen im rechten Fuss, dessen Sprunggelenk geschwollen und druckempfindlich ist. A.usserdem besteht Oedem beider unteren Extremitäten.

10. Mai. Der Kranke fragt "ob er hier nicht in Hamburg sei?" schrieb einen zusammenhängenden Brief an seine Frau und erholt sich auch körnerlich zuséhends.

30. Mai. Die Besserung schreitet regelmässig fort, dabei fehlt die Erinnerung für die Zeit der letzten Erkrankung vollständig; er weiss, dass er wegen Gelenkleidens in's Krankenhaus gekommen, aber er kann sich des Friedrichsberg und der Reise nach Schleswig nicht errimnern.

Im Juni erschien der Kranke psychisch ganz frei, war äber das Krankhafte seines Zustandes ganz klar nnd arbeitete fleissig in der Tischlerwerkstatt. Dabei war das rechte Sprunggelenk noch geschwollen und etwas schmerzhaft und diese Schwellung hielt noch im Juli an, obschon so unbedeutend, dass Patient ohne Beschwerden gehen konnte. Im August wurde er geheilt entlassen und kehrte nach Hamburg so wohl zurück, dass er seine Arbeit als Schiffszimmermann wieder aufnehnen konnte.

Doch nur auf kurze Zeit. Schon im November zeigte sich wieder Dyspnoe, Mitte December stellte sich Husten und Appetitlosigkeit ein, Anfangs Januar 1873 schwollen die Füsse an, es traten Kopfschmerzen, öfteres Erbrechen auf und am 24. Januar $1873 \mathrm{kam}$ er wieder in das allgemeine Krankenhans, (Abtheilung des Herrn Dr. Enge1-Reimers).

Hier warde neben Herzhypertrophie und Ergüssen in die Pleurahöhlen ein bedeutender Eiweissgehalt des Urins und einzelne helle Cylinder in demselben beobachtet und die durch die Sektion vollkommen bestätigte Diagnose 
Die Geistesstörungen im Verlaufe des akuten Gelenkrheumatismus. 669

auf Schrumpfniere gestellt. Der Kranke delirirte, knirschte mit den Zähnen und ging in wenig Tagen zu Grunde.

Die Sektion ergab vollständige Verwachsung des Herzens mit dem Herbeutel, Herzhypertrophie ohne Klappenfehler, wandständige Thromben an der Spitze des linken Herzens und im rechten Herzohr, kleinere hämorrhagische Infarcte der Lungen.

Das Gehirn ergab starke Trübung und Verdickung der Pia, mässige Erweiterung der Ventrikel und Granulationen des Ependym derselben bei starker Anämie der Hirnsubstanz. Endlich fanden sich, wie schon erwähnt, exquisite Schrumpfnieren.

Ich habe die letzte Erkrankung nicht ausführlicher mitgetheilt, weil uns hier nur das Bild des Nierenleidens, resp. der urämischen Affektion entgegentritt.

Dieses Nierenleiden hat offenbar schon lange bestanden, ohne Symptome zu machen. Das Fehlen des Eiweiss bei der Untersuchung in Schleswig beweist nichts dagegen, da bekanntlich bei Schrumpfniere öfter das Eiweiss auf ein Minimum reduzirt ist oder ganz schwindet.

Die Geistesstörung war eine melancholia cum stupore. anfangs so. intensiv, wie ich selbst keinen Fall gesehen, so dass ich den Kranken anfangs für soporös bielt und an urämische Intoxication oder eine organische Hirnerkrankung dachte.

Die Besserung der geistigen Verstimmung scheint hier mit dem Wiederauftreten der Affektion des rechten Sprunggelenkes zusammenzufallen.

\section{Fïnfter Fall. (XXVI. Beobachtung).}

Akuter Gelenkrheumatismus. Fndooarditis und Pneumonia duplex. Im Beginn der Reconvalescenz Melancholie von ca. 6! monatlicher Dauer. Kein Reeidiv der Gelenkaffektion. Heilang bei bleibender Aortenstenose.

Heinrich Gr., 25 jähriger Schuhmachergeselle aus Niendorf, wurde, nachdem er bereits fast $\delta$ Tage za Hause krank gelegen, am 24. Februar 1872 wegen Rheumatismus artic. acutus in die Klinik zu Kiel anfyenommen.

Der Verlauf der Gelenkerkrankungen wax ein sehr gelinder, die Fiebererscheinungen nicht ungewöhnliche. Dabei zeigten sich jedoch sehr bald endocarditische Lrscheinungen - deren Endresultat eine geringe Stenosirung der Aortenklappen zu sein scheint - und beiderseitig Verdichtungen in den hinteren Lungenpartien. Patient war während dieser Zeit stets bei voller Besinnung, war anfangs zwar häufig etwas verzagt, später jedoch guten Muths. Nach am 16. März erfolgter Abfieberung bemerkte man noch nichts auffallendes in seinem Benehmen. Am 17. Abends zeigte er sich zuerst närrisch und weigerte sich zu essen. Ant 18. bereits ausgeprägte Melancholie. In den folgenden Nacht schlief Patient nach einer Dosis ron $0,05 \mathrm{Grm}$. Morphium 
durchaus nicht, gegen Morgen jedoch einige Stunden lang nach $3,0 \mathrm{Grm}$. Chloralhydrat. Erwacht zeigte er sich viel munterer, antwortete auf Fragen und ass mit gutem Appetit. Mittags war er jedoch bereits wieder in dumpfes Hinbrüten verfallen, aus dem er bis jetzt nicht wieder erwacht ist. $\mathrm{Zu}$ weilen hatte er Hallucinationen beängstigender Natur, oft so lebhaft, dass er in ängstliches Wimmern und entsetztes Schreien ausbrach. Zuw Essen, Trinken, Uriniren etc. muss er stets dringend aufgefordert werden; einmal wurde die Applikation des Catheters nöthig. Schlaf erfolgte nur nach Chloral (3-6 Grm.). Die Temperatur des Kranken blieb fast normal, erreichte nur einige Male 38,20. Patient verliert an Körpervolumen.

In diesem Zustande wurde er der Irrenanstalt zu Schleswig überwiesen, wo am 18. Mai folgender stat. praes. aufgenommen wurde:

Patient sitzt still und verzagt fast den ganzen Tag auf einem Fleck, spricht gar nicht, nur mit grosser Mühe und nach wiederholtem Fragen antwortet er leise und ängstlich höchstens "ja" oder "nein“. Er will nicht essen, thut es jedoch von selbst, wenn er dazu aufgefordert wird.

Körperlicher Zustand: Klein und schmächtig gebaut, abgemagert und blass, wiegt 48,5 Kilogramm bei einer Körperlänge von $160 \mathrm{Cm}$. Schädel symmetrisch. Lungen gesund, auch hinten ist der Ton überall gleichmässig hell und hört man überall vesiculäres Athmen. Herzdämpfung im Längsdurchmesser vergrössert. Herzstoss nach aussen von der Mamillarlinie. Beide Töne über der Aorta unrein.

Ordinirt: Ruborirende Diät. Acid. phosph. Bäder.

30. Mai. Wird etwas freier, spricht nur selten, geht auf eignen Wunsch mit auf Gartenarbeit.

Juni. Ist recht fleissig bei der Arbeit, macht noch einen ängstlichen verzagten Eindruck, erholt sich körperlich.

Juli. Die Besserung schreitet fort, der Kranke räumt sein krankhaftes Verhalten ein, weiss keine Gründe dafür anzugebeo.

A u gust. Unveränderte Fortdaner des besseren Zustandes. In der letzten Woche des Monats mehrere unregelmässige Fieberanfille, die nach Chin. mur. 0,6. dos. 2, schwanden.

September. Erscheint psychisch ganz frei, ist stets in gleichmässiger zufriedener Stimmung, behauptet nach wie vor nicht angeben zu können, weshalb er nicht habe sprechen und essen wollen.

ok to ber. Genesen entlassen.

\section{Sechster Fall. (XXVIr. Beobachtnog).}

Azuter Gelenkrheumatlsmus mit Endo- und Pericarditis. Moroses Wesen. Plötzlicher Ausbruch der Geistesstörung im Beginn der Reconvalescenz. Wechsel von Melancholie und Manie, dann albernes schwachsinniges Benehmen.

Heilung nach sechsmonatlicher Dauer der Geisteskrankheit.

[Mai 1855:] Heinrich Chr. Br., gehürtig vom Gute Bothkamp, hatte, nach-dem er bereits in anderén Orten des Herzogthums als Geselle gearbeitet, seit dem Herbste des verflossenen Jahres in Kicl in Arbeit gestanden und war während dieser Zeit von seinem Meister in seinen Arbeiten brauchbar, in 
seinem sonstigem Benehmen vernünftig und erost befunden worden. Er erkrankte in der ersten Hälfte des Februar d. J. zufolge einer heftigen Erkältung, die er sich auf der Heimkehr von einem Tanzvergnügen zugezogen hatte, an vagem, febrilen Gelenkrheumatismus und wurde des genannten Uebels wegen am 15. Februar in das akademische Krankenhaus zu Kiel aufgenommen. Die rheumatischen Schmerzen zeigten sich unter fortdauerndem, mässigen Fieber bald in den Knie-, bald in den Schulter-, bald in den Handgelenken. Der Kopf des Kranken war dabei vollkommen frei; er zeigte in seinem geistigen Wesen, mit Ausnahme einer zuweilen auffallenden, morosen Wortkargheit, durchaus keine Abnormität. Ausser den Schmerzen in den Gelenken war es indess eine selbst durch Opiate nicht zu beseitigende Schlaflosigkeit, tuber welche der Kranke mehrfach und dringend klagte. Während sich in den ersten 10 Tagen seines Aufenthaltes im Lazarethe Symtome einer Complication mit entzündlicher Herzaffektion bei wiederholien Untersuchungen nicht herausgestellt hatten, wurden an 25. Februar in wahruehmbaren Herzgeräuschen die ersten Spuren endocarditischer und pericarditischer Exsudationen am Herzen bemerkt, indessen auch diese Zeichen einer entzündlichen Affektion des Herzens schwanden allmählig unter angemessener Behandlung gleichzeitig mit den vorhingenannten rheumatisclien Gelenkschmerzen. Das Fieber hatte sich gänzlich verloren, der Schlaf und Appetit kehrten zurück und der Kranke schien einer vollkommenen Reconvalescenz mit Sicherheit entgegen zu gehen, als sich am 5. März, nachden er bei der Visite noch ruhige und vernünftige Antworten, wie immer, gegeben hatte, ganz plötzlich die Erscheinungen einer Geistesverwirrung einstellten, insofern der Kranke bei ganz ruhigem, selbst langsamem Pulse und kühlem Kopfe unter der wiederholten, lauten Klage „er sei verloren, er müsse sterben", ganz plötzlich das Bett zu verlassen rersuchte und nur mit grösster Mühe in demselben zurùckzuhalten war. Zu dieser melancholischen Gemüthsverstimmung mit 'geistiger Verwirrung gesellten sich im Laufe der folgenden Tage und Wochen manialalische Anfälle, in denen der Kranke bald unter lautem Weinen, bald unter ebenso unmotivirtem Lachen, seine Kleider, sein Bett zerstörte und an seinem Wärter sich thätlich vergriff, so dass die Anwendung äusserer Zwangsmittel bei demselben nothwendig wurde. Im Laufe der nächstfolgenden Zeit indess schwanden unter dem inneren Gebrauche des Tart. stibiat and protrahirter lauwarmer Bäder mit kalten Umschlägen auf den Kopf und kräftiger Ableitung auf die äussere Haut die Erscheinungen dieser krankhaften Ueberreizung des Geistes. Dagegen blieben die Symptome einer geistigen Verwirrung, welche nach oft längeren freien Intervallen besonders dann aufzutreten schienen, wenn der Kranke sich von Anderen beobachtet glaubte. Bei vielfacher, körperlicher Bewegung im Freien, augemessener Diät und möglichst luhiger Isolirung des Kranken sind auch diese Spuren periodischer Geistesverwirung immer seltener geworden und der Kranke war namentlich in der letzten Zeit schon so weit gekommen, dass er den Zustand seiner früheren Verwirrung selbst anerkannte und dass er, der früher ganz unbekümmert und planlos in den Tag hinein lebte, nun von freien Stïcken den Wunsch, zu seinen früheren Beschäftigungen zurückzukehren, äusserte, auch in einem veruünftig geschriebenen Briefe an seinen Vater einen ziemlich klaren Bericht über seinen eigenen Krankheitszustand und seine Genesung gab. Dem ungeachtet traten auch in der letzten Zeit noch immer 
unverkennbare Zeichen einer plötzlichen angstrollen Unruhe und Verwirrung der Gedanken bei ihm deutlich hervor und es wurde daher, da es zur vollkommenen Wiederherstellung seiner Gesundheit nothwendig erschien, dass er unter Verhältnisse gebracht wuide, die, neben der ärztlichen Beaufsichtigung, ihm die Möglichkeit einer zweckmässigen Beschäftigung und Thätigkeit gewährten, der Antrag auf die Uebersiedelung des Kranken in die Irrenheilanstalt zú Schleswig gestellt.

Es darf für die genügende Beurtheilung des Kranken nicht unerwähnt bleiben, dass derselbe gerade um die Zeit, als er von seiner rheumatischen Krankheit genesen war, die Aufforderang erhalten hatte, sich zur Untersuchung wegen des Militairdienstes zu stellen.

Dem mir vorliegenden Physikats-Gutachten, welches die Anfangs-Erkrankung als Nanie bezeichnet, entnehme ich folgende Angaben über das Befinden des Kranken Ende April:

"Gegenwärtig ist nur noch zurück eine zuweilen an Narrheit grenzende Gemüthsschwäche, welche sich durch dann urd wann geäusserte alberne Reden und Handlungen zu erkennen giebt, indess auch ron Tage zu Tage mehr und mehr schwindet. So hat er vor Kurzem auf dern Rande seines Bettes sitzend, plötzlich einige Schwenkungen mit den Armen gemacht und ausgerufen: „nu sit ick up de Locomotiv"; ein ander Mal fragt er den Secundärarzt bei dem Anblicke eines Reiters, ob sie nicht zusammen ausreiten wollen; als dieser es rerneint, sagt er: „nu kann man keen Ritter sien, so wullen wi ers en Töller vull Grütt äten". - Neulich ist er Nachts aufgestanden, um ein Geschäft zu verrichten, hat dann aber die Nachtlampen ausgelöscht."

Dieses Verhalten des Kranken, verbunden mit einem häufig wiederkehrenden albernen Lächeln, bezeichnet das Attest ganz treffend als „an Narrheit grenzende Gemüthsschwäche".

Der Kranke wurde in die Anstalt nach Schleswig versetzt, wo er sich, wie das Nachfolgende ergiebt, bald vollständig erholte.

Nachdem er in den ersten Tagen seines Schleswiger Aufenthalts ruhig und in sich gekehrt erschienen war und in seinem Benehmen etwas Scheues an den Tag gelegt hatte, leitete sich bald ein besinnlicherer Zustand ein. Er fägt sich willig der Hausordnung, verhält sich ruhig und vernünftig, beschäftigt sich fleissig im Garten, spricht besonnen und zusammenhängend, räumt seine Krankheit ein, freut sich berzlich seiner Genesung und verspricht dieselbe hier ruhig abwarten zu wollen.

Körporlicher Zustand: Geringe Abmagerung, schlanker graciler Körperbau, regelmässige Schädelbildung, blondes Haar, blaue Iris, bewegliche Pupille, chronische Augenentzündung, besonders des rechten Auges mit Schwund der Wimpern, schlanker Hals, gewölbter Brustkasten, regelmässige Brustorgane bis auf eine grössere Dämpfung in der Herzgegend. Normale Unterleibsorgane. Schlaf gut. Puls etwas frequent. Aussehen blass. wasser.

Ord.: Leichte gute Diät. Warme Bäder. Acid. phosphor, später Bitter-

1855, Juli. Geistiges und körperliches Wohlbefinden.

August. Geheilt entlassen. 
Der Krankheitsfall ist besonders beachtenswerth durch das Her. vortreten geistiger Schwäche, eines albernen Wesens, welches die eigentliche Geistesstöruog Monate lang überdauerte. Es ist dies einerseits ein interessantes Analogon zu ähnlichen Fällen geistiger Schwäche nach Typhus, andererseits entspricht es ganz dem Verlanf der Erkrankung in unserer XXIV. Beobachtung.

\section{Siebenter Fall. (XXVIII. Beobachtung).}

Akuter Gelenkrheumatismus. In der Reconvalescenz Manie mit melanoholischen Perioden. Heilung nach 8 Monaten.

Carl Rudolph Rohde aus Flensburg, Schiffer, $21 \mathrm{Jahr}$, wurde am 24. März 1848 wegen Manie in die Irrenanstalt zu Schleswig aufgenommen. Derselbe erkrankte im September 1847 auf der Reise nach St. Thomas an Gelenkrheumatismus. Nach der Anwendung von reizenden Ableitungen besserte sich auf St. Thomas das körperliche Befinden, worauf er nach dem Abgange von St. Domingo in einen unruhigen Zustand mit grosser Besinnungslosigkeit verfiel, der in der letzten Zeit mit einer weinerlichen Stimmung sich complicirte und stets mit anhaltender Leibesverstopfung verbunden war.

\section{Status bei der Aufnahme.}

Grosse Aufregung, Neigung zu Gewaltthätigkeit, allgemeines Delirium mit grosser Ideenverwirrung, Unreinlichkeit. Von Mitte Juni an trat eine allmählige Besserung ein, er wurde besinnlicher, sprach besonnen und fing an zu arbeiten. Diese Besserung war nach einem Zeitraum von 5 Wochen in Genesung übergegangen, so dass der Kranke am 24. Juli geheilt entlassen wurde.

\section{Achter Fall. (XXIX. Beobachtung).}

Akuter Gelenkrheumatismus. INelancholie, im Anfang mit Krämpfen (Chorea,?). Heilung nach ca. $4 \frac{1}{2}$ monatlicher Dauer.

H. Behrens, Handschubmacher aus Rendsburg, 28 Jahr alt, wurde wegen Melancholie am 1. Juli 1852 in Schleswig aufgenommen. Derselbe war wegen Gelenkrheumatismus behandelt, seit Anfang Juni tiefsinnig, klagt über Gedankenlosigkeit, Schwindel, spricht von seinein Ende. Bald darauf zeigten sich Krämpfe in den Extremitäten und dem Unterkiefer, Herzklopfen, Schlaf- und Sprachlosigkeit, hierauf Irrereden und Neigung zu verkehrten Handlungen. Erbliche Anlage fehlt. Ais Ursache wird zu bänfig ausgeübter Coitus angegeben.

\section{Status praesens bei der Aufnahme.}

Juli. In dem stillen ruhigen Benehmen, dem schleichenden Gang, dem fremdartigen Blick spricht sich schon die Alienation seiner Geisteskräfte aus. Seine mit leiser Stimme und langsamer Sprache geführten Reden offenbaren Aengstlichkeit und Furcht. Er fühlt sich sehr krank und ist, wie er selbst 
sagt, hierhergekommen, um durch Bäder gesund zu werden. Er verlangt sebr nach Arzenei.

Körperlicher Zustand: Grosse Abmagerung und Schwäche.

Ord.: Zuerst warme Bäder - später Regenbäder -, innerlich Decoct. chïn. mit Acid. phosph. Nahrhafte Diät. Beschäftigung im Garten.

September. Das kórperliche Befinden hat sich sehr gebessert. Patient hat sehr zugenommen. Die Aengstlichkeit und Furcht haben nachgelassen, doch treten noch zuweilen Wahnvorstellungen hervor, z. B. dass Henker und Richter hier im Hause seien und er deshalb nicht gesund werden könne. Verlangen nach Hause. Therapia eadem.

26. Oktober. Geistig und körperlich vollständig wohl. Genesen entlassen.

Ich habe in der summarischen Uebersicht dieses Falles die "Krämpfe in den Extremitäten und Unterkiefer" als vermuthliche Choreaartige Bewegungen bezeichnet. Jedenfalls müssen dieselben bald vorübergegangen sein, da in Schleswig keine Störungen der Motilität bemerkt wurden. Auch in diesem Falle trat kein Recidir der Gelenkaffektion und doch baldige Heilnng der Geistesstörung ein.

Ich schliesse diesen Krankengeschichten eine kurze Uebersicht der sämmtlichen mir aus der Litteratur bekannten Fälle an, da eine Zusammenstellung derselben nirgends gegeben ist und die grosse Mehrzahl derselben, die von $T u ̈ n g e l$ und mir veröffentlichten, welche über die Hälfte des gesammten Materials umfassen, sich in wenig verbreiteten Werken finden.*)

1) Griesinger I. Frau, 30 Juhr. Im zweiten Wochenbett Puerperalmanie. Rheumat. acut. Im Beginn der Reconvalescenz Geistesstörung, Anfangs Erregung, dınn Melancholia cum stupore mit Erregungszuständen wechselnd. Oeftere Recidive der Gelenkaffektion ohne wesentlichen Einfluss auf den Verlauf, beim ersten Rccidiv Uebergang von maniakalischen Zuständen zu Melancholia cum stupore. Heilung nach 2 Monaten.

2) Mesnes (arch. génér. 1856. I. 711. Griesing er II.). Mann, 23 Jahro alt, aknter Gelenkrhenmatismus mit Pleuritis, gegen Ende der Krankheit maniakalischer Zustand, choreaartige Bewegungen. Wiederholte Recidive der Gelenkaffektion ohne Einfluss auf die Geistesstörung. Heilung nach 3 Monaten.

*) Von Tüngel's Arbeit findet sich in Schmidt's Jahrbüchern CXVI. p. 48 (1862) ein ausführliches Referat, welches aber nur die Beobachtungen akuter nervöser Zufälle auf der Höhe der Krankheit einzeln vorfübrt und die Fälle mit Geistesstörung nicht weiter berücksichtigt. Meine erste grössere Arbeit ist in Schmidt's Jahrbüchern gar nicht referirt, wohl aber im II. Bd. des Virchow-Hirsch'schen Jahresbericht für 1866 und im med. Centralblatt 1866 p. 90 bis 94, beide natürlich ohne Mittheilung der Casuistik. Von dem Nachtrag findet sich eine sehr ausfuhrliche Besprechung $H$ uppert's in Schmidt's Jahrbilchern OXLVIII. p. 324. (1870). 
3) Flemming (Pathologie und Therapie der Psychosen p. 88, Griesinger III). Frau, 30 Jahre. Erschöpfung. Akuter Gelenkrheumatismus. Melancholia attonita. Heilung, Dauter?

4) Delioux (Archives génér. 1857. I. 670, Griesinger VII.) Mann, $21 \mathrm{Jahr}$, Soldat. In der Reconvalescenz einer nur 4 Tage dauernden Gelenkaffektion plötzlich Geistesstörung (Melancholia). Entlassung im Beginn der Reconvalescenz.

5 bis 7) Ulrich I.-III, junge Männer (Pericarditis 1 mal), Melancholie von 8, 12, 12 Tagen. (Deutsche Klinik 1859. p. 263).

8) Burrows I. (Beobachtungen über die Krankheiten des cerebralen Blutkreislaufes und den Zusammenhang zwischen Hirn- und Herzleiden. Deutsch von Posner, Leipzig und Wien 1847. Fall IX. p. 146, bei Griesinger IV.*) Frau, 24 Jahr, sehr schwere Gelenkaffektion, Pericarditis, Delirien, aus diesen sich allmählig entwickelnde Melancholie. Nach 2 Monaten ungeheilt entlassen.

9) Burrows II. (ibid. Fall $X$, bei Griesinger V.) Fran, 14 Jahr. Ob Herzleiden? Bei'm Bestehen der Gelenkaffektion Geistesverwirrung und heftige Bewegungen (Chorea?), Melancholie, nach einem Monat ungeheilt.

10) Burrows III. (ibid Fall XI. p. 149). Frau, 18 Jahr. Endocarditis. In der Reconvalescenz plötzlich ausbrechende Melancholie. Heilung nach 12 Monaten.

11) Burrows IV. (ibid. Fall XII.) Mann, 17 Jahr. Sehr protrahirter Rheumatismus. Grosse Erschöpfung. Endocarditis. Melancholischer Zustand. Recidiv der Gelenkaffektion ohne Einfluss auf das psychische Verhalten. Tod. Section des Hirns ergiebt nichts besonderes.

*12) Tüngel I. (1857, Klinische Mittheilungen von der medicinischen Abtheilung des allgemeinen Krankenhauses zu Hamburg aus dem Jahre 1858, Hamburg 1860. p. 41, bei Griesinger VI). Mann, 20 Jahr. Peri- und Endocarditis. In der Reconvalescenz Melancholie, dabei „wie in geringem Grade blödsinnig". Patient später (jedenfalls erst nach Jahren) ganz wohl. (Simon, Charité-Annalen XIII. p. 118).

13) Tüngel II. 1861. (XV. der nervösen Zufülle im akuten Gelenkrheumatismus in den klinischen Mittheilungen etc vom Jahre 1860. Hamburg 1862. p. 99-132). Mann, 33 Jahr. Zweiter Anfall ron Rheumatismus acut. Keine Herzaffektion. Bei Nachlass der Gelenkaffektion Melancholie mit zweimaligem Recidiv der Gelenkaffektion, jedesmal Verschlimmerung der Melancholie, ,zitterte bei Bewegungen, Sprache etwas stammelnd". Heilung.

14) Tüngel III, 1860 (ibid. Fall XVI.) Frau, 21 Jahr. Herzaffektion. Gegen Ende aes Rheumatismus Geistesstorung, Melancholie. Heilung nach ca. $11 / 4$ Monat.

15) Tüngel IV. 1860. (ibid. XVII) Mann, 18 Jahr. Endocarditis. Pleuritis duplex. Melancholia agitata während Bestehen des Pleura-Exsudats. Heilung nach ca. 11/2 Monaten.

16) Tüngel V. 1861. (ibid. XVIII.) Fran, 18 Jahr. Endocarditis mit Be-

*) Griesinger citirt zu Burrows I. und II. die Gazette méchicale de Paris 1843. p. 800, offenbar ein Referat über das Werk Burrows. 
klemmung, allmählig deutliche Melancholie. Erfolglose Opiumbehandlung. Heilung nach ? Dauer.

17) Tüngel VI. (ibid XIX.) Frau, 19 Jahr. Peri- und Endocarditis. Melancholie im Beginn der Reconvalescenz. Heilung nach ca. 14 Tagen.

18) Tüngel VII. (ibid XX.) Mann, 24 Jahr. Pericarditis. Pneumonie. In der Reconvalescenz Melancholie mit Dementia-ähnlichem Zustand wechselnd. Variola. Heilung nach ca. 3 Monaten.

19) Tüngel VIII. (ibid XXI.) Frau, 36 Jahr. Endocarditis. In der Reconvalescenz Melancholia agitata, plötzlich ausbrechend. Nach 41/2 Monat ungeheilt abgegangen.

20) Tüngel IX. (ibid XXII.) Frau, 42 Jahr. Pleuritis duplex. Peri- und Fndocarditis, Verwirtheit, sich zur Tobsucht steigernd. Allmählige Besserung in ca. $11 / 2$ Monaten. Recidiv der Gelenkaffektion mit sofortiger Verschlimmerung der psychischen Symptome. Tod nach 1 Monat. Autopsie mit negativem Hirnbefund.

21) Tüngel X. (ibid. XXIII.) Mann, 17 Jahr. Peri- und Endocarditis. Besserung. Fieberloses Recidiv der Gelenkaffektion mit melancholischer Verwirrtheit, ,automatische Bewegungen wie bei Chorea, grimassirte beständig". Tod nach 8 Tagen. Section: negativer Hirnbefund.

*22) Peyser I. (über die protrahirte Form der rheumatischen Hirnaftektion. Berliner Inangural-Dissertation von 1867). Mann, 15 Jahr. Melancholische Verstimmung, akuter Gelenkrbeumatismus. In dessen Verlauf Melancholie mit einer plótzlichen Erregung beginnend. Sehr protrahirter Verlauf, ,schien zuletzt in der Anstalt fast schwachsinnig zu werden, so dass die Prognose für sehr bedenklich befunden wurde", ist aber später gänzlich geheilt und nach 3 Jahren noch ganz wohl gewesen. (Privat-Mittheilung des Herrn Dr. Wilh. Sander).

23) Peyser II. (ibid. p. 22). Mann, $42 \mathrm{Jahr}$, vor 14 Jahren Rheumatismus acut. mit nachfolgender Geistesstörung, dann 2 Erkrankungen ohne solche, jetzt im 4. Anfall Herzaffektion und Geistesstörung (Melancholie). Besserung mit Eintritt eines Recidiv der Gelenkaffektion, dann geistige Schwäche, Verwirtheit bei bestehender Gelenkaffektion. Entlassung in beginnender Reconvalescenz nach ca. 1 Monat.

24) Rosenthal. Knabe von 16 Jahren. Am Herzen systolisches Geräusch. Im Beginn der Reconvalescenz Melancholie von ca. 1 monatlicher Dauer. Heilung. (Aus Wiener Medicinalhalle IV. Jahrgang 1863. p. 153 in E. O. Flamm, über meningitische Symptome von Rheumatismus akutus. Tübinger Inaugural-Dissertation 1865. p. 25).

25) Mugnier. (Folie consécutive aux maladies aigues. Paris 1865, p. 57). Fran, $32 \mathrm{Jahr}$, ohne hereditäre Anlage, aber tuberculös. Ob Herzaffektion fraglich. Melancholie bei Bestehen der Schmerzen. Heilung nach 11/2 Monat.

26) Besser. (Zeitschrift für Psychiatrie XXII. p. 253). Mann (Soldat), 23 Jahr alt. Sehr schwerer Rheumatismus, Blutverluste per nares et annm. Ob Herzaffektion fraglich. Bei Nachlass der Schmerzen Melancholie. Chorea. Heilung nach $71 / 2$ Monat.

27. Simon I. (1864). Mann, 16 Jahr. Erysipelas faciei, akuter Gelenkrheumatismus und Pleuropneumonie. Nelancholie mit Ausbruch der Pneumonie beginnend. Heilung nach $31 / 2$ monatlicher Dauer. 
28) Sim on II. (1860). Frau, 24 Jahr. Beginn der Melancholie mit Eintritt des Rheumatismus acutus. Entlassung im Beginn der Reconvalescenz nach 5 wöchentlicher Dauer.

29) Simon III. (1861). Frau, 21 Jahr. Sehr intensiver Gelenk-Rheumatismus einer scrofulösen hysterischen Person. Keine Herzaffektion. Melancholie ron 21/2 Monate Dauer, beginnend bei noch bestehender Gelenkaffektion. Heilung.

30) Simon IV. (1863). Frau, 23 Jahr. In der Reconvalescenz Melancholie von 1 monatlicher Dauer. Heilung.

31) Simon V. (1863). Frau, 21 Jahr. Rheumatismus acutus mit Pericarditis. Starke Anämie. Recidiv der Gelenkaffektion. In der Reconvales. cenz Melanchclie von 2 Monate Dauer. Heilung.

32) Simon VI. (1864). Mann, 24 Jabr. Rheumatismus acutus mit Recidiv und grosser Anämie, In der Reconvalescenz Melancholie, gleichzeitig leichte Variola. Nach 3 Wochen gebessert entlassen. Ausgang unbekannt.

33) Simon VII. (1865). Frau, 19 Jahr. Rheumatismus acutus fast aller Gelenke mit Peri- und Endocarditis, nach 9 Tagen Aufhören der Gelenkaffektion, dann Recidiv mit Pleuritis sinistra. Melancholie mit maniakalischen Anfällen und Temperatursteigerung. Tod anter heftigen Convulsionen. Sektion: Blutreichthum des Hirns, Verwachsung des Herzens mit dem Herzbentel.

*34) Simon VIII. (1865). Frau, 21 Jahr. Intensiver akuter Gelenkrheumatismus mit Herzaffektion, während des Bestehens der Gelenkschmerzen Melancholie. Dreimaliges Recidiv der Gelenkaffektion, jedesmal mit Ver * schlimmerung der Melancholie. Heilung nach 5 monatlicher Dauer. War 1870 auf meiner Abtheilang im allgemeinen Krankenhause mit Insufficienz der Mitralis im Stadium gestörter Compensation. Geistig vollkommen klar. Starb im Mai 1870, nachdem sie die letzten 8 Tage delirirt. Sektion: Ganz frische Pachymeningitis und Insufficienz der Mitralis.

35) Simon IX. (1863). Mann, 20 Jahr. Rheumatismus acutus mit Herzaffektion und Pleuritis. In der Reconvalesceuz Melancholia simplex. Recidiv der Gelenkaffektion ohne Einfluss auf den Zustand. Heilung nach 3 Wochen.

36) Simon X. (1862). Frau, $22 \mathrm{Jahr.} \mathrm{Rheumatismus} \mathrm{acutus} \mathrm{mit} \mathrm{Peri-}$ carditis. Im Beginn der Reconvalescenz Melancholie mit maniakalischen Unterbrechnngen. In der Besserung Recidiv der Gelenkaffektion mit Verschlimmerung der Stimmung. Heilung nach 14 Tagen.

37) Simon XI. (1860). Frau von 35 Jahren. Im ersten Wochenbett Puerperal-Manie, im dritten akuter Rheumatismus. In der Reconvalescenz Melancholie. Recidiv der Gelenkaffektion ohne Einfluss auf den Geisteszustand. Heilung nach 1 Monat.

38) Simon XII. (1863). Frau, $33 \mathrm{Jahr}$. Sehr intensiver Rheumatismus acutus. Herzaffektion; im Beginn der Reconvalescenz Melancholie. Recidiv der Gelenkaffektion ohne Einfluss auf die Melancholie. Allmählige Besserung. Heilung nach 3 monatlicher Dauer.

39) Simon XIII. (1863). Frau, 20 Jahr. Rheumatismus acutus mit Pericarditis. Melanchulie mit Verfolgungsideen nach dem ersten Recidiv der Gelenkaffektion. Bedeutende Besserung. Mit dem zweiten Recidiv coincidirt einc beträchtliche Verschlimmerung der Melancholie. Nach 2 Monaten nicht vollständig geheilt entlassen. 
40) Simon XIV. (1866). (XIX. Beobachtung des "Nachtrag" Charité-Annalen Bd. XV. p. 124). Mann, 22 Jahr. Rheumatismus acutus. Peri- und Endocarditis. Rechtsseitige Pleuropneumonie. Auftreten von Melancholie während des Bestehens der Gelenkaffektion, letztere überdauernd. Anfänglich Opiumbehandlung. Heilung nach 5 Monaten.

41) Simon XV. (1866). (XX. Beobachtung ibid.), Frau, 20 Jahr. Hereditäre Disposition und Anämie im Pnerperalzustand. Alruter Gelenkrheumatismus. In der Reconvalescenz plötzlich eintretende Melancholie, im Beginn mit Erregungszuständen. Systolisches Geräusch am Herzen, Heilung nach 2 Monaten. Erkrankt nach 2 als Puella publica verlebten Jahren an fieberbaftem Magenkatarrh, dasach Geistesstörung von ca. 5 Monaten Dauer und Heilung.

Hierzu kommen dann als

42 bis 45 ) (Simon XVI-XIX) die in dieser Arbeit mitgetheilten Beobachtungen XXII-XXV und als 46 bis 49) (Rüppell I-IV) die aus den Schleswiger Akten ausgezogenen Beobachtungen XXVI-XXIX.

50) Wille I. (Zeitschrift für Psychiatrie XXIII (1866) p. 105). Frau, 32 Jahr. Hereditäre Anlage. Rheumatismus acutus mit „unerträglichen" Schmerzen. Auf der Höhe der Krankheit schwinden Anschwellung and Schmerzhaftigkeit der Gelenke, dafür "heitere Exaltation mit grosser Verwirtheit". Endocarditis. Chorea. Allmühliger Nachlass d's Fiebers. Uebergang der Verwirtheit in Melancholie. Reconvalescenz nach 1 Monat. Recidiv der Gelenkaffektion mit psychischer Verstimmung. Heilung nach 1 Monat.

51) Willo II. Frau, 42 Jahr. Schweres Trauma des Schädels im 22. Jahre. Anfälle von Kopfschmerz, Krämpfen, Delirien, Insufficienz der Mitralis. Melancholia activa. In der Reconvalescenz Rheumatismus acutus. Schwinden der Gelenkaffektion, Ausbruch einer intensiven Melancholia cum stupore. Wiederauftreten der Gelenkaffektion ohne Einfluss auf die psychische Störung. Ischias. Heilung nach ca. 2 Monat. (L. c. p. 107.)

52) Wille III. (ibid. p. 110). Mann, 19 Jahr. Schreibekrampf. Rheumatismus acutus mit ungeheuren Schmerzen. Recidiv mit Fieber, Krämpfen, psychischer Erregung, Symptome von Verdichtung der Lungenspitzen und Endocarditis. Choreaartige Bewegungen. Tobsucht, dann Stumpfheit, Dementia. Salivation. Auftreten ron Gelenkschmerzen ohne Einfluss auf den Verlauf. Heilung nach ca. 2 Monaten.

53) Sander I. (Rheumatismus and Geisteskrankheit. Zeitschrift für Psychiatrie Bd. XX. p. 216). Soldat, 21 Jahr. Rheumatismus acutus; bei noch bestehendem Fieber und Gelenkaffektion plötzlich ausbrechende Melancholie. Uebergang in Stupiditat, sehr gesunkene Ernährung. Anfangs systolisches Geräusch. Später Schwäche der unteren Extremitäten, dann Fieber. Heilung nach ca. 7 Monaten.

*54) Sander II. (ibil. p. 219). Soldat, 22 Jahre, etwas imbecill. Rheumatismus acutus. Melancholie zu tiefem stupor sich steigernd. Allmählige Besserung. Entlassung im Zustand geistiger Stumpfheit, (letzteres nach Besser Zeitschrift für Psychiatrie XXII. p. 252).

55) (Aus dem Bayerischen ärztlichen Intelligenzblatt 1866. Nr. 33 bis 35 in Virchow's Jahresbericht für 1866. II. p. 2). Soldat. Rheumatismus acutus 
Die Geistesstörungen im Verlaufe des akuten Gelenkrheumatismus. 679

mit Endocarditis. In der Reconvalescenz Mania religiosa, übergehend in Melancholia cum stupore. Ausgang?

56) Ledru, (Gazette des hôpitanx 1867. Nr. 94). Soldat, 21 Jahr. Rheumatismus acutus mit 2 maligem Recidiv, Peri- und Endocarditis, Manie, in stille Delirien übergehend. Tod nach 1 monatlicher Geistesstörung durch Perforation eines Psoas-abszess in die Bauchhöhle. Sektion: hochgradige HirnAnämie.

57) Diego Coco, (Klinik von Tomasi) [aus Jl Morgagni 1866. VIII. 5. p. 389 in Schmidts Jahrbücher CXLIX p 154]. Mädchen, 18 Jahr. Akuter Gelenkrheumatismus. Hochgradige Anämie. Pericarditis. Lungenkatarrh. Oedem. Allmäblige Besserung. Plötzlich in der Nacht ein Anfall höchster Angst mit Delirien, dann Bewusstlosigkeit, neue Pericarditis, Ausgang in Melancholie (Vergesslichkeit, Schweigsamkeit, trauriges, stilles Wesen).

Hierza kommen die im Antang besprochenell Arbeiten, nämlich:

58 und 59) Cloust on I. und II.

*60 und 61) Ferber I. und II., endlich

62) Christian.

Ich habe in dieser Zusammenstellnng diejenigen Fälle, welche ich durch Angaben aus der Litteratur oder Privat-Mittheilungen ergänzen konnte, mit einem Stern bezeichnet. Ton den früher von mir aufgerechneten Fällen habe ich 'T üng' el XIV der akuten nervösen Symptome und Simon XIV der Geistesstörungen im Verlauf des akuten Gelenkrheumatismus fortgelassen; wenn man aber auch noch mehrere der hier aufgezählten 62 Fälle kassiren wollte - und ich kann nicht lengnen, dass sich gegen manche, z. B. Sim on VII, Peyser I, Will e II Einwürfe erheben lassen - so bleibt immerhin noch die stattliche Zahl von mehr als einem halben Hundert, welche nach mancher Richtung hin die ron Griesinger auf Grundlage von 7 Fällen gegebene Darstellung ergänzen und erweitern lässt.

Die Zusammenstellung der Daten über Alter und Geschlecht lasse ich bei Seite, da sie meiner Ueberzengung nach, wenn ans einem so bunt zusammengewürfelten Material genommen, keinen Werth hat.

Viel wichtiger ist die Frage nach der Frequenz der Erkrankung, die sich in zwei Theile zerlegen lässt: wieviel Prozent der Geistesstörungen sind durch akuten Gelenkrheumatismus bedingt und wie viel Prozent der akuten Gelenkrheumatismen werden durch Geistesstörung complicirt. Die Antworten auf die erste Frage lauten sehr verschieden. Christian*) giebt an, dass in Montdevergues von 1860-1872 ca. $2000^{\circ}$ Kranke aufgenommen seien, davon 30 nach akuten Krankheiten. Von diesen 30 waren 11 Typhen, dagegen wie es scheint kein

*) L. j. c. 
einziger Rheumatismus-Fall. *) $\mathrm{Za}$ ganz anderen Resultaten führen die Schleswiger Zahlen, welche wir in dem ,summarischen Bericht über die Irrenanstalt bei Schleswig" von J. Rüppel1**) finden. Danach waren unter 2893 Geisteskranken, die von 1835-1870 aufgenommen worden, nicht weniger als 15 in Folge akuten Gelenkrheumatismus erkrankt, und zwar von 1512 Männern 12, von 1381 Frauen 3.

Diese Zahlen, nach denen für etwas über $1 / 2 \%$ der sämmtlichen Irren Rheumatismus als Ursache der Erkrankung anzusehen wäre, stehen in befriedigender Uebereinstimmung mit den Hamburger Beobachtungen.

Von 2195 auf der Irrenstation des allgemeinen Krankenhauses und dem Friedrichsberg behandelten, resp. abgegangenen Kranken aus den Jahren 1865-1871 sind 6 Rheumatismusfälle, also etwas über $1 / 4 \% * * *$ )

Da diese beiden Zahlenreihen gat stimmen, da sie eine ansehnliche Krankenzahl und einen zusammenbängenden längeren Zeitraum umfassen, da die beiden Anstalten fast die ganze Krankenmasse ihrer resp. Gebiete erhalten und diese Gebiete selbst unmittelbar aneinander grenzen, so kann man die Zahl von ca. ein halb Procent für die nordalbingischen Lande als gutbegründet nehmen.

Die kleine Differenz zu Gunsten Schleswigs dürfte sich unschwer aus dem Bestreben der Kieler Kliniken, ihre Kranken nicht zu lange zu behalten, erklären, während in Hamburg einzelne Fälle Monatelang anf der medizinischen Abtheilung verblieben.

Sieht man sich andererseits nach Material um zur Erledigung der noch interessanteren, weil für die Prognose der akuten Gelenkrheumatismen wichtigen Frage: anf welche Zahl von Rheumatismus art. acutus eine Geistesstörang falle - so wird man auf wunderbare Widersprüche stossen.

Kreusser fand im Stuttgarter Catharinenhospital unter 305 akuten Gelenkrheumatismen keinen einzigen Fall chronischer Geistesstörung $\dagger$ ),

*) Der von ihm mitgetheilte Fall stammt aus der Elsässer Irren-Anstalt Stephansfeld.

**) Hamburg 1872. p. 37 .

***) Die den jährlichen Berichten entlehnten Zahlen sind 350, 307, 337, 369, 301, 379, 252. Die Zahl pro 1865 im Bericht für 1866. Die früheren Berichte sind, da die Delirium tremens Kranken mit eingerechnet sind, für unseren $Z$ weck nicht $z u$ verwerthen, sonst würde noch eine grüssere Uebereinstimmung mit den Schleswiger Ziahlen erzielt werden.

f) Vir c how - Hirs ch, Jahresbericht für 1866. II. 269. Aus würt. Corresp. Blatt 1866. No. 14, 
Die Geistesstörungen im Verlaufe des akuten Gelenkrheumatismus. 681

ebensowenig Scholz im Bremer Krankenhanse unter $80^{*}$ ) von 18681871 (April) Aufgenommenen; in den älteren Bremer Krankenhausberichten finde ich unter 289 von 1857-1865 aufgenommenen Fällen **) kein einziges Mal Geistesstörung and nur einmal „Hirnhaut-Entzündung6***) als Complication aufgeführt.

Ein noch schlagenderes Zeugniss für die Seltenheit der Geistesstörung nach akutem Gelenkrheumatismus legen die Wiener Krankenhausberichte mit ihren gewaltigen Zahlenreihen ab.

In den Abhandlungen des Wiedener Krankenhauses für 1866, 18681870 finde ich unter über 600 Rheumatismus acutus Fallen 71 Fälle mit Herzerkraukungen aber keinen mit Geistesstörung complicirt. $\dagger$ )

Unter circa 1500 Fällen, die in den Jahren 1857-1860, 1865, 1866, $1868 \mathrm{im}$ allgemeinen Krankenhause zu Wien an Rheumatismus articul. acutus erkrankten $†$ ) weist nur ein einziger (ans dem Jahre 1866) die Complication mit Melancholie auf, während nicht weniger als 335 Complicationen seitens des Herzens beobachtet wurden.

Rechnet man noch hinzu, dass unter den 62 von mir zusammengestellten Fällen ron Irresein in Folge akuten Gelenkrheumatismus nur ein einziger (Nr. 24) aus Oesterreich stammt, so wird man zu dem Ergebniss geführt, dass in Wien Geistesstörung eine so seltene Complication der in Rede stehenden Krankheit sei, dass unter 2000 Rheumatismen kaum einer Geisteskrank wird.

Zu ganz anderen Ergebnissen führt die Statistik des Hamburger allgemeinen Krankenhauses, $\dagger$ †), welche für mehr als 1\% aller Rheu-

*) Geisteskrankheiten aus Anämie. Archiv für Psychiatrie Heft 3 des III. Bandes p. 732 .

**) Die den "ärtlichen Berichten" entlehnten Zahlen sind: 15, 25, 28, 28, 57, 21, 36, 50, 59; davon mit Herzkrankheiten complicirt 1857-1863: 6, 8, $2,8,8,13$.

***) 1. c. Bericht für 1859 .

†) Die den äratlichen Jahresberichten entlehnten Zahlen sind ron mir folgendermassen zusammengestellt - cfr. die folgende Anmerkung - 1866: 137 mit 12 Herzcomplicationen, 1868: 110 mit 20, 1868: 256 mit 26, 1870: 156 mit 13.

†) Die Wiener Berichte stellen akute und chronische Fälle, Muskel- und Gelenkrheumatismen zusammen. Circa der dritte Theil ist, laut Angabe des 57., 59. und 60. Berichtes anf den akuten Gelenkrheumatismus zu beziehen. Für 1865, 66, 68 habe ich die akuten Fälle berechnet. So ergeben_sich folgende Zahlen (die Herzcomplicationen in Klammern): 1857 ca. 240 (35), 1858 ca. 300 (52), 1859 ca. 230 (41), 1860 ca. 260 (37), 1865 ca. 256 (40), 1866 ca. $319(62), 1868$ ca. 259 (68).

†††) Nach den "ärztlichen Berichten" zusammengestellt, die Fälle von Geistesstörung nach Tüngel's und meinen Arbeiten. 
matismus-Fälle die schwerwiegende Complication der Geistesstörung ergiebt; aber auch diese Statistik stimmt mit anderen Thatsachen: der, dass die Hälfte des überhaupt vorliegenden Materials aus Hamburg stammt wie der, dass unter den in Schleswig und Hamburg aufgenommenen Geistesstörungen eine verhältnissmässig so grosse Zahl als durch Rheumatismus bedingt aufgeführt wird. (s. o.)

\begin{tabular}{|c|c|c|c|c|c|c|}
\hline \multirow{2}{*}{$\begin{array}{l}\text { Jahr- } \\
\text { gang. }\end{array}$} & \multicolumn{3}{|c|}{ Rhenmatismus-Fälle. } & \multicolumn{3}{|c|}{$\begin{array}{c}\text { Davon erkrankten an } \\
\text { Geistestörung. }\end{array}$} \\
\hline & Männer. & Frauen. & Summa. & Männer. & Frauen. & Summa. \\
\hline 1858 & 73 & 56 & 129 & - & - & - \\
\hline 1859 & 71 & 36 & 107 & 一 & - & - \\
\hline 1860 & 58 & 33 & 91 & 1 & 1 & 2 \\
\hline 1861 & 59 & 39 & 98 & 1 & 2 & 3 \\
\hline 1862 & 69 & 54 & 123 & - & 1 & 1 \\
\hline 1863 & 74 & 60 & 134 & 1 & 3 & 4 \\
\hline 1864 & 103 & 50 & 153 & 2 & - & 2 \\
\hline 1865 & 89 & 42 & 131 & - & 2 & 2 \\
\hline $1866 *)$ & 61 & 39 & 100 & 1 & 1 & 2 \\
\hline 1867 & 77 & 33 & 110 & - & - & - \\
\hline 1868 & 102 & 66 & 168 & 1 & - & 1 \\
\hline $\left.1869^{* *}\right)$ & 34 & 23 & 57 & - & - & - \\
\hline $1870 * * *)$ & 32 & 21 & 53 & - & - & - \\
\hline \multirow[t]{2}{*}{1871} & 60 & 63 & 123 & 1 & - & 1 \\
\hline & 962 & 615 & 1577 & 8 & 10 & 18 \\
\hline
\end{tabular}

Wir finden demnach unter 1577 Fällen ron akutem Gelenkrheumatismus, die im allgemeinen Krankenhause zu Hamburg behandelt worden, 18 Fälle ron Geistesstörung, also etwas über 1 Prozent, und zwar

$$
\begin{aligned}
& \text { unter } 962 \text { bei Männern } 8=\text { ca. } 0,8 \% \\
& \text { unter } 615 \text { bei Frauen } 10=\text { ca. } 1,6 \%
\end{aligned}
$$

d. h. die Complication war bei Frauen noch einmal so häufig als bei den Männern.

Auffallig ist die verschiedene Betheiligang der verschiedenen Jahre an der Zahl der Kranken - ein Umstand, der dringend zur Benutzung sehr grosser Zahlenreihen bei solcher Statistik auffordert. Zähle ich zu den Krankenhausfällen die direkt in die Irrenanstalt aufgenommenen

*) Die Statistik dieses Jahres findet sich im ärztlichen Bericht von 1867.

**) Die Zahlen beziehen sich nur auf die 2. Hälfte des Jahres.

***) Es fehlt ein Theil - etwa der vierte, da die IV. medizinische Abtheilung keine Statistik geliefert. 
und die Fälle ans der Privat-Praxis von Ferber und mir, so kommen für Hamburg auf die Jahre 1858, 1859, 1867 and 1869 kein Fall, 1857, 1862, 1870 bis 1873 je 1 Fall, 1864 bis 1866 und 1868 je 2 Fälle, 18613,18604 und 1863 sogar 5 Fälle.

Ich füge hier, um mit den statistischen Angaben zum Abschluss zu gelangen, die spärlichen Zahlen bei, welche ich über die Zahl der Rhenmatismus-Geisteskrankheiten im Vergleich zu den durch andere akute Krankheiten bedingten auffinden konnte.

Die Schleswiger Statistik über 2993 Fälle giebt als KrankheitsUrsache an:

\begin{tabular}{|c|c|c|c|c|c|c|}
\hline Typhus & & i 21 & Männern, & 29 & Frauen, & Summa \\
\hline Rheumatismus acutus & , & 12 & " & 3 & $"$ & $n$ \\
\hline Intermittens & $"$ & 4 & $"$ & 1 . & $"$ & " \\
\hline Scharlach & " & 3 & " & 1 & $"$ & $"$ \\
\hline Cholera & $\eta$ & 2 & $"$ & 1 & , & "Y \\
\hline Variola & ( & - & " & 1 & , & " \\
\hline
\end{tabular}

Christian, der die französische Litteratur sehr vollständig gesammelt hat, die deutsche aber offenbar nur sehr unvollständig kennt, zählt 114 Fälle ron Geistesstörung nach aknten Krankheiten auf, nämlich 36 nach Typhus, 17 nach Pneumonie, 16 nach akuten Exanthemen, 16 nach Intermittens, 13 nach akutem Gelenkrheumatismus, *) 9 nach Cholera, 2 nach Gesichtsrose und 3 andere.

Der Güte des Herrn Dr. Reye verdanke ich noch folgende Angaben:

Unter, den vou 1869 bis 1873 in Friedrichsberg, resp. von der Irrenstation des allgemeinen Krankenhauses abgegangenen Kranken fanden sich aus der Kategorie der nach akuten Krankheiten alienirt gewordenen: 36 und zwar

nach akutem Gelenkrheumatismus 12 (event. 14, aber der eine Fall gehört wahrscheinlich zum Typhus, ein anderer den Isactations-Psychosen auf hereditärer Basis an),

nach Ileotyphus 16 ,

nach Intermittens 2,

nach Scarlatina 2,

nach Angina diphtheritica 1,

nach Cholera 3 (alle aus dem Jahre 1873).

Zum Vergleich gebe ich die Zahl der puerperalen Affektionen. Die-

*) Davon 6 Männer und 7 Frauen unter überhaupt 68 Männern und 46 Frauen. 
selbe war nicht kleiner als 86 , übertraf also die Gesammtsumme der Psychosen nach akuten Krankheiten um mehr als das Doppelte.

Höchst auffällig - und ganz abweichend von den Zahlen anderer Autoren, ist bei uns das Verhältniss der Typhus-Alienationen zu dem der Rheumatismus.Psychosen, um so bemerkenswerther, als die Zahl der Typhen im allgemeinen Krankenhause die der Rheumatismen stets um das Doppelte, oft um das 3-4 fache übersteigt. Geistesstörungen nach Pneumorie finden sich nicht auf unserer Liste - sie verliefen so rasch, dass sie im Krankenhause verblieben.

Wenden wir uns ron diesem Versuche, die ersten Grundlagen für eine Statistik der Rheumatismus-Geistesstörungen aufzustellen, za der Frage, welche dem Studium dieser Erkrankung ein besonderes Interesse verleiht, zur Diskussion darüber: ob die Geistesstörungen, welche im Verlaufe des akuten Gelenkrheumatismus auftreten, verschieden sind vun den nach anderen akuten Krankheiten entstehenden, ob sie als eine Theil-Erscheinung des Rhenmatismus, eine „protrahirte rheumatische Hirnaffektion" (Griesinger) angesehen werden müssen, - so haben wir zu ihrer Erledigung eine Reihe von Punkten zu erörtern, die in ihrer Gesammtheit die ganze Pathologie der uns beschäftigenden Erkrankung umfassen, und die wir dahin präzisiren können:

welches sind die Formen, in denen die Geistesstörung nach akutem Gelenkrheumatismus auftritt?

ist die Art der Erkrankung, die sie begleitenden Erscheinungen, der Verlauf, die Prognose, der anatomische Befund verschieden ron denen, wie wir sie bei Geistesstörungen nach anderen akuten Krankheiten finden?

I. Die Form der Geistesstörung. Es ist behauptet worden, die Form der Psychose sei bei den nach akutem Gelenkrheumatismus entstandenen psychischen Affektionen verschieden ron denen nach anderen akuten Krankheiten. Schon Griesinger deutet dies an, besonders präguant aber spricht Peyser es aus: „die überwiegend meisten der berichteten Fälle haben die psychologische Form der Melancholie. Diese Erscheinung findet sich nicht bei den durch Anämie bedingten Hirnaffektionen nach anderen Krankheiten; hier findet sich vielmehr hä̈ufig nur Verwirrtheit, die in die verschiedensten psychologischen Formen übergehen kann."*) Aber diese letztere Angabe ist entschieden nicht richtig, wie schon ein Blick auf die Zusammenstellung Christian's lehrt. Denn dieser fand unter den 114 Geistesstörungen

*) L. j. c. p. 8. 
nach akuten Krankheiten (worunter nur 15 Rheumatismusfälle) als häufigste Erkrankungsformen Manie bei 34, Melancholie bei 16 und den angeblich gerade für Rheumatismus so charakteristischen Stupor sogar in 27 Fällen!

Unsere Uebersicht der Rheumatismus-Fälle zeigt, dass die Geistesstörung in sebr verschiedenen Formen auftreten kann. Wir finden neben den (überhaupt als primäre Formen am hänfigsten auftretenden) Melancholien auch Manien und Formen von Dementia. Bei der Prüfung der von uns beobachteten oder zusammengestellten Krankheitsfälle nach Rheumatismus treten aber besonders deatlich and mit grösseren Zahlen drei Formen geistiger Störung hervor: die Melancholia cum stupore, eine eigene Art alternirenden Irrsinns und eine der akuten Dementia anzareihende Form von Narrheit.

Die Melancholia cum stupore (mel. attonita) ist nicht selten von vornberein zugegen; andere Male entwickelt sie sich schnell aus einen mehr maniakalischen Zustand heraus. Ein ganz vorzügliches Beispiel. ist meine $\mathrm{XXV}$. Beobachtung, weil bei ihr der Stupor in solcher Intensität und so anhaltend auftrat, dass ich anfangs an urämischen Sopor und dann an tiefe Dementia dachte. Und wenige Monate nachher ging der Kranke vollständig geheilt aus Schleswig ab!

Aber diese Melancholia cum stupore ist durchaus nicht charakteristisch für Rhenmatismus-Psychosen.

Ich habe sie wiederholt nach Typhus gesehen und in der Statistik Christian's zeigt sie sich neben der Manie als die häufigste Form.

Weit eher könnte man die von mir als ,alternirende" bezeichnete Form für charakteristisch halten, doch auch sie findet sich ausser nach akuten Krankheiten auch bei hereditärer Anlage, insbesondere bei einzelnen „hysterischen" Irrsinnsfällen.

Es besteht diese Erkranking in einem raschen Wechsel verschiedener primärer Formen, insbesondere der Manie und Melancholie, untermischt mit Dementia-ähnlichen Zuständen. Von der Folie circulaire unterscheidet sie sich durch das rasche, ganz unbestimmte und, unbestimmbare Wechseln ohne die Zwischenstufe eines relativ normalen Zustandes; von der Melancholia agitata dadurch, dass in ihr die aktive Haltang, die Gewalthätigkeit nicht durch das zur Explosion führende Uebermass innerer Beängstigung hervorgerufen wird, sondern wirklich eine „expansive" Stimmung obwaltet. Zwischendurch tritt auch ,eine mehr demente Verwirrtheit" auf.

In diese Kategorie gehört der erste Musterfall von Rheumatismus- 
psychosen, der einzige damals von Griesinger selbst beobachtete (Nr. 1 meiner Zusammenstellung), von meinen Fällen insbesondere $X$ (Nr. 36), XXIII, XXIV in dem ersten Theil seines Verlanfes, XXYII und XXVIII, ferner der Christian'sche Fall und auch wohl Wille I und III (Nr. 50 und 52). Beobachtung XXIII. zeigt deutlich, dass auch ganz kurze Störungen in dieser Form verlaufen können.

Die dritte von mir erwähnte Form ist eine eigenthümliche zwischen Tobsucht und Dementia stehende Verwirtheit, ein albernes kindisches Wesen, das man am ehesten als Schwachsinn bezeichnen kann. Ich habe schon gelegentlich der XXIV. und XXVII. Beobachtung auf sie hingewiesen, bei welchen beiden sie den Uebergang von der alternirenden Geistesstörung zur Heilung vermittelt; auch Peyser II (Nr. 23), Tüngel VII (Nr. 18) und Sander II (Nr. 50) gehören hierher, vielleicht auch Peyser I in seinem späteren Verlauf.

Ich will noch auf eine dem Gebiet der" "Monomanien" oder Dementia zuzutheilende geistige Störung kurz hinweisen. Bekanntlich kommen Typhasfälle vor, bei denen die Patienten einzelne Wahnideen oder Reihen von Wahnvorstellungen festhalten, während sie in allen übrigen Beziehungen klar und verständig erscheinen. Ich habe mich in einem Fall von einen Patienten durch solche Monomanie vollständig täuschen lassen. Obschon ich an die Möglichkeit derselben dachte, erschien mir der. Kranke bei einer genauen Prüfung seiner geistigen Fähigkeiten so vollständig klar, dass ich seiner Angabe, ihm sei Geld gestohlen, vollständig Glauben schenkte, zumal er einzelne charakteristische Stücke genau beschrieb. Erst am folgenden Tage, als eine neue, als solche sofort klar zu erkennende, Wahnidee auftauchte, sah ich meinen Irrthum ein. Unter unseren Rheumatismus-Fällen ist diese Art der Alienation nirht vertreten, allein ich weiss durch freundliche Mittheilung des Herrn Professor W estph al, dass diesem eine hierher gehörige Beobachtung auch nach akuten Gelenkrheumatismus bekannt ist.

Auffällig ist in unserem Verzeichniss die geringe Zahl ron Maniefällen, von denen noch obendrein kein einziger eine ganz reine Manie darstellt. Aber auch nach Typhen sind reine Manien sehr selten, sie finden sich mehr nach kurz verlaufenden, schnell abfallenden Erkrankungen: Pneumonien, akuten Exanthemen, Erysipelen.

Kurz - in der Art der geistigen Störung, welche sich im Verlauf des Rhenmatismus art. acutus entwickelt, liegt Nichts, was von den nach anderen akuten Krankheiten, insbesondere dem Typhus, entstandenen abweicht, nichts für die Rheumatismus-Fälle Charakteristisches. Sehen wir zu ob in 
Die Geistesstörungen im Verlaufe des akuten Gelenkrheumatismus. 687

II. dem Verlauf derselben etwas Besonderes, ihnen allein Zukommendes liegt. Wir hätten hier zunächst das Verhältniss zwischen Geistesstörung und Gelenkaffektion und das Auftreten der Chorea zu besprechen.

Schon Mesnet, einer der ältesten Beobachter, spricht von einen Alterniren zwischen Gelenkaffektion und Geistesstörung, ohne dass nach der Angabe Griesingers selbst - aus seinem Falle (Nr. 2) dies klar hervorgeht. Auch in Griesinger's, eigenem Falle findet bei den Recidiven der Gelenkaffektion keine Besserung, nur eine Formänderung der Geistesstörung statt. Dennoch spricht sich Griesinger für das Alterniren aus, vorsichtiger in seinem Aufsatze, bestimmter im Lehrbuch. ${ }^{*}$ ) Ich habe aber schon früher**) in einer ausführlichen Besprechung der einschlagenden Fälle gezeigt, dass nicht selten ganz im Gegentheil das Recidiv der Gelenkaffektion sofort eine bedeutende Verschlimmerung resp. Wiederausbruch der Geistesstörung hervorruft und ich brauche daher nur auf meinen Fall VIII zu verweisen, der an sich genügt, um alle Theorien, die auf einen Wechsel zwischen Psychose und Gelenkaffektion begründet sind, umzustossen.

Drei Thatsachen haben meiner Ueberzeugung nach vorwiegend dazu beigetragen, einen grossen Theil der Aerzte dieser Theorie geneigt zu machen und einzelne Beobachter irrezuführen.

Einmal tritt die ausgesproshene Geistesstörung meist in der Reconvalescenz, also nach Aufhören der Gelenkaffektionen ein. Allein sehr häufig ist dies nicht der Fall, eine Veränderung im geistigen Verhalten tritt oft schon auf der Höhe der Krankheit ein, oder die Alienation schliesst sich an Delirien an. Zuweilen ist ron Beginn der Krankheit an die geistige Störung nachzuweisen, so in Christian's Fall und in der hier mitgetheilten XXIV. Beobachtung. Selbst in den Fällen, in welchen die Geistesstörung scheinbar ganz akut in der Reconvalescenz ausbricht, sind psychische Abnormitäten schon vorher bemerkt, so bei Sander I (Nr. 53), meiner XXIII. und der (Schleswiger) XXVII. Beobachtung.

Ferner trifft öfter mit dem Wiederauftreten der Gelenkaffektion eine Aenderung, aber keine Besserung der psychischen Affektion ein, die Form ändert sich, die Störang bleibt. Findet diese Aenderung in der Form eines Uebergangs von tobsüchtiger Verwirrtheit zu stillen melancholischen Wahnideen statt, wie bei Griesinger Nr. 1 und in

*) 2. Auflage p. 194.

**) Charité-Annalen XIII. 2. p. 120. 199.

Archiv f. Psychiatrie. IV. 3. Heft. 
meiner XX[V, Beobachtung, so kann mau leicht die grössere Rahe für den Beginn der Besserung halten, wie dies mir selbst gerade in dem XXIV. Fall anfangs erging.

Ein dritter Grund zu Tauschungen liegt in der Art der Gelenkaffektion bei Rheumatismos acutus. Nicht selten ist der Erguss in die Gelenke so gering, dass, während die Kranken über die intensivsten Schmerzen klagen, objektiv nichts nachweisbar ist. Geht in einem solchen Fall das Bewusstsein verloren, so liegt kein Beweis für das Fortbestehen der Gelenkaffektion vor, und man wird um so mehr geneigt sein, an ein Verschwinden derselben zu glauben, wenn man Kranke in tobsüchtiger Erregung dieselben Gelenke auf das Heftigste bewegen sieht, welche sie kurz vorher auf das Sorgsamste vor jeder Bewegung hüteten. Aber auch hier zeigt der schon öfter citirte Kranke XXIV, wie wenig das bei ihm objektiv nachweisbare Fortbestehen der Gelenkafiektion einen tobenden Kranken im Gebrauch der Gelenke hindert.

Im Ganzen berechnet Peyser das Verhältniss der Fälle, in denen der Wiedereintritt der Gelenkaffektion von günstigem Einfluss, von ungünstigem Einfluss und von keinem merklichen Einfluss war, wie $5: 5: 5$; ich kann nach wiederholter Prüfung der einschlagenden Fälle es höchstens als 2:7:8 angeben. Wie übrigens das Verbältniss sich stellt, jedenfalls wird man die vereinzelten Beobachtungen eines günstigen Einflusses des recidivirenden Gelenkleidens nicht als wesentlich für die rheumatische Psychose auffihren können. Und ganz das gleiche gilt von der Complication mit Chorea. Wir finden dieselbe überhaupt nur in 11 Fällen, also nur in ca. $18 \%$ aller Beobachtungen, und unter diesen 11 sind 3 (Nr. 9, 13, 21, 49) immerhin zweifelhaft, unter den übrigen 7 sind 2 Kinder und ein junger Mann, der schon vorher Chorea gehabt. Trotz dieser Einschränkung lässt sich die Thatsache nicht wegleugnen, dass ,chorea-artige" Bewegungen nicht ganz selten die Geistesstörung nach akutem Gelenkrheumatismus compliciren. Ich sage mit Absicht "chorea-artig", weil eine genaue Schilderung der Bewegungsstörungen fehlt und doch erst eine genaue Analyse die Bewegungen der Chorea von ataktischen und Schüttelbewegungen abtrenuen lässt. Dass aber chorea-ähnliche Motilitätsstörungen auch nach anderı akuten Krankheiten vorkommen, haben gerade in der letzten Zeit die wichtigen Arbeiten Westphal's für Typhus und Variola,*) und im Anschluss daran Scheper**) für Masern erwiesen. Dass die Rheu-

*) Dieses Archiv III. p. 376 .

**) Berliner Klinische Wochenschrift 1872. Nr. 43. 
Die Geistesstörungen im Verlaufe des akuten Gelenkrheumatismus. 689

matismus-Fälle häufiger mit Bewegungsanomalien vom Charakter der Chorea complicirt sind, ist ja so bekannt, dass man in Frankreich darauf ein ganzes Gebäude von Theorien begründet hat, und wenn beim Gelenkrheumatismus im Ganzen die Chorea nicht in der Häufigkeit von $18 \%$ anftritt, wie in unseren Fällen, so muss man bedenken, dass diese anch ihre besondere Disposition zu Nervenstörungen gerade durch den Eintritt der Geisteskrankbeit erwiesen haben.

Mehrere der Rheumatismus-Choreafälle sind mit Herzaffektion complicirt gewesen, andere im Anfang der Krankheit nicht beobachtet, so dass die Herzaffektion nicht ausgeschlossen ist; dass aber zu Herzleiden sich Chorea gesellt, auch ohne dass Rheumatismus zugegen, ist eine bekannte Thatsache. *)

Chorea-artige Bewegungen compliciren demnach die RheumatismusPsychosen, finden sich aber auch nach andern akaten Krankheiten, sind in nur $1 / 6$ der Rheumatismusfälle zugegen, und bilden also keinen wesentlichen Zug in der Erscheinung der nach akutem Gelenkrheumatismus auftretenden Geistesstörung.

Ich will im Anschluss an die chorea-artigen Bewegungen noch anf andere, unsere Fälle complicirenden Motilitätsstörungen hinweisen.

Schon im XXI. Fall der "nervösen Zufälle" beschreibt Tüngel bei einer nach Rheumatismus geisteskrank gewordenen Frau einen kataleptischen Zustand: „sie lag regungslos, liess den aufgehobenen Arm längere Zeit in der Luft schweben, die gebildete Stirnfalte schwand langsam, die Respiration war schwach und langsam; bein Berühren der Angenlider zeigte sich indessen Reflexbewegung in diesen.... Sie verharrte in diesem Zustande bis zur Zeit des Abendessens, wo sie dann die Augen anfschlug und mit gehörigem Appetit ass. Das Esseu musste ihr aber in den Mund gebracht werden." Einen ähnlichen Zustand habe ich in meiner XXIV. Beobachtung geschildert. Die beiden Fälle zeigen zugleich eine andere Aehnlichkeit: motorische Störungen in Form continuirlich fortgesetzter Bewegungen, „die Kranke fing an, fortdauernd Pendelbewegungen zu machen, theils von einer Seite zur andern, theils von hinten nach vorn" heisst es bei Tüngel, und auch mein Patient XXIV. machte halbe Stundenlang ein und dieselbe Bewegung: Kopfschütteln, Klopfen der Bettdecke mit beiden Händen u. dergl.

Ferner finden wir in dem I. Fall Clouston's, Sander's und

*) Ich verweise z. B. auf den Fall Abercrombie's bei Burrows l.j.c. p. 142, weil hier kein Klappenfehler, sondern Pericarditis die Chorea hervorrief. 
Ferber's motorische und sensible Lähmung resp. Formicationen und Contracturen der unteren Extremitäten.

Endlich sei im Anschluss an die atactischen Symptome des Stammelns gedacht, welches in Tüngels XV. Fall der "aervösen Zufälle" auftrat, und zeitweilig auch bei dem oft erwähnten Kranken XXIV. bemerkt wurde. Bei letzteren machte es auf mich stets den Eindruck des Intendirten, zumal er dabei öfter den Namen eines etwas stotternden Freundes nannte.

Es erübrigt uns noch, die häufigsten Complicationen unserer Fälle, die Affektionen des Respirations- und Circulations-Apparates kurz zu erwähnen.

Pneumonien nnd Pleuritiden sind ebenso häufig vertreten wie die Chorea und mehrfach als direkte Todesursache anzusehen, z. B. in dem Ferber'schen I. und meinem VII. Fall. Besonders intensiv sind die Complicationen seitens des Respirations-Apparates im XXIII. Fall, in welchem wir 2 mal Pleuritis und 1 mal Pneumonie, alle 3 linkerseits, zu beobachten hatten.

Herzkrankheiten im weiteren Sinne (d. b. Peri- und Endocarditis) sind bei den Geistesstörungen nach Rhenmatismus so hänfig, dass Burrows in einen Fall, in welchem keine Symptome der Pericarditis im Bartholomew's Hospital hatten anfgefunden werden können, dennoch eine solche annimmt: „Obgleich in diesem Falle die physikalischen Zeichen einer entzündlichen Herzaffektion nicht aufgefunden wurden, so kann über das Vorhandensein einer solchen kein $\mathrm{Z}_{w}$ eifel obwalten, wenn man die ganze Krankheitsgeschichte dieser Patientin" (Nr.9 unserer Zusammenstellung), ,mit anderen ähnlichen Nervenleiden vergleicht, welche sich im Verlanfe des Rhenmatismus einstellen und denen dann nur jene insidiöse Krankheit zu Grunde liegt."*) Ich habe schon früher gezeigt, $\left.{ }^{* *}\right)$ dass, in dieser Starrheit ausgesprochen, der Burrows'sche Satz nicht haltbar ist, dass unbezweifelbare Beobachtungen vorliegen, in denen keine Herzaffektion nachgewiesen war. Ich kann mich daher hier kurz fassen, und das Ergebniss meiner Uebersicht der beschriebenen Fälle aufstellen:

Dass ausgesprochene Herzaffektionen (Peri- und Endocarditis) in mehr als der Hälfte aller Fälle ausdrücklich hervorgehoben werden, dass in einer Reihe anderer Fälle die Beobachtung keine vollständige war, dass aber auch in einer allerdings geringen Anzahl von Erkrankungen die Complication mit Herzaffektion auszuschliessen ist.

*) L. j. c. p. 148.

**) Charité-Annalen XIII. p. 127, XV. p. 150. 
Dies führt zu dem Schlusse, dass in der Herzkrankheit als solcher die Ursache der Rheumatismus-Psychose nicht gefunden werden kann, legt aber ebenso die Vermuthung nahe: dass in der Mehrzahl der Fälle die Herzaffektion die zur Erzeugung der Psychose günstigen Bedingungen - wie ich*) schon früher anseinandergesetzt, und nenerdings Scholz*) bestätigt, Anämie des Hirns - setzt.

Man könnte gegen die den Herzaffektionen beigelegte Bedeutung den Einwand erheben, dass so viele Kranke trotz bestehen bleibenden Herzfehlers von ihrer Geistesstörung geheilt werden und bleiben (cfr. unsere VIII. Beobachtung). Allein Herzfehler bedingen an sich noch keine Anämie des Gehirns, vielmehr ist gerade die Zeit des Einbruchs eines Klappenfehlers mit den gewaltigen Circulationsstörungen, die er nach sich zieht, die für Blutgehalt und Ernährung des Gehirns gefährlichste Zeit, während sich allmählig durch Herzhypertrophie die nöthige Compensation ausbildet.

Ich habe hier noch einen andern, den Circulationsverhältnissen im Hirn scheinbar sehr fernliegenden Punkt zu erwähnen, die Schmerzen des akaten Gelenkrheumatismus. Es war dem praktischen Scharfblick Tüngel's nicht entgangen, dass die Schmerzhaftigkeit der Gelenkaffektion in der Erzeugung der Psychose eine Rolle spiele.***) „Ich habe stets solche Kranke mit akutem Gelenkrheumatismus, welche nicht aufhörten zu klagen und zu jammern, denen die Hitze, der Schweiss die Unbeweglichkeit unerträglich waren, mit grosser Sorge betrachtet, da mir bei solchen Individuen einige mal jene plötzlich auftretenden Zufälle vorgekommen waren" sagt er später betreffs der schweren akuten Hirnzufälle. $\dagger$ ) Wir finden in der Casuistik der RheumatismusPsychosen nicht wenig Fälle, in denen die Gelenkaffektion, sei es besonders ausgedehnt, sei es besonders langwierig oder ganz aussergewöhnlich schmerzhaft war.

Zur Erklärung können wir die wichtigen Experimental-Untersuchungen Nothnagel's †') heranziehen, wonach Erregung peripherer Nerven, insbesondere des Cruralis anf reflektorischem Wege Verengerung der Piagefässe hervorruft, und zwar besonders intensiv bei Erregung der Nervenendigungen. Dass aber mit Verengerung der Piagefässe Anämie des Gehirns, und zwar gerade der Grosshirnrinde bewirkt wird, muss

*) Charité-Annalen XIII. p. 127-132.

**) L. j. c. p. 747 .

***) Klinische Mittheilungen für 1860. Hamburg 1862. p. 101.

†) Ibid. p. 120.

††) Virchow's Archiv XL. p. 203-213, 
jedem, der die Anordnung der Blutgefüsse dieses Gebietes kennt, klar sein.*)

Wenden wir uns vom Verlauf und der Complication III. zur P rog$\mathrm{n} o$ se, so ist auch betreffs dieser für die Rheumatismus-Psychosen eine eigene Stellung insofern beansprucht worden, als ,die Genesung da am schnellsten und sichersten" erfolgen soll, ,wo nach einiger Zeit im Verlauf der Hirnstörung wieder die Gelenke rheumatisch befallen werden" (Griesinger).

Auch zur Prüfung dieses Satzes giebt nnsere Zusammenstellung jedem Leser das Material zur Hand. Sie zeigt uns:

1) dass die Prognose der Rheumatismus-Geistesstörungen eine vorzügliche ist, dass die überwiegende Majorität der Fälle sicher geheilt ist, einige wenige im Zustand beginnender Reconvalescenz oder noch ungeheilt abgingen, dass als ungeheilt konstatirte Fälle eigentlich nicht existiren. Hierin schliesst sich die Rheumatismus-Psychose ganz der nach Pneumonie, Erysipel u. s. w. an und unterseheidet sich von der nicht ganz so günstigen, die im Typhus auftritt;

2) dass die Mehrzahl der Fälle in 2 bis 4 Monaten günstig verlaufen, nicht selten die Geistesstörung noch kürzere Zeit, circa 14 Tage anhält;

3) dass man aber selbst nach einem Verlauf von mehr als 4 Monaten, nnd ebenso nach Eintritt scheinbar ungünstiger Symptome (eites Dementia-ähnlichen Zustandes) die Hoffnung nicht aufaugeben braucht und

4) dass Eintritt und Nichteintritt von Gelenkaffektion oder von Chorea ohne jeglichen Einfluss auf die Prognose sind.

Die günstige Prognose erklärt es auch, warum IV. die pathologis ch-anatomischen Befunde so selten sind. Die Zahl der sezirten Fälle ist sehr gering, die vorhandenen Sektionsberichte geben in Betreff des Hirns einen ganz negativen Befund, da auf Blutfülle oder Blatarmuth der Hirngefässe die den Tod bedingende Complication, hier zumeist Herz- und Langenaffektion, von entscheidendem Einfluss ist. Es muss daher als unberechtigt zurückgewiesen werden, dass Flamm in seiner unter Köhler's Auspicien in Tübingen verfassten, übrigens sehr fleissig gearbeiteten Dissertation $\mathbf{T}$ üngel aus dessen eigenen Sektionsprotokollen zu widerlegen, dass er den Beweis zu führen versucht, dass auch in Tüngel's Fällen der Befund kein

*) Cfr. Heubner, Ernährungsgebiet der Hirnarterien. Medicinisches Centralblatt 1872. p. 817 . 
negativer, sondern Meningealhyperämie sei*) und von einer Lokalisation des Rheumatismus in den Meningen spricht.**)

Im Uebrigen halte ich den Beweis, dass wirklich im Gehirn keine naehweisbaren Veränderungen vorkommen, für noch nicht geführt, da bisher gar keine genaueren mikroskopischen Untersuchungen vorliegen es ist mir aber a priori sehr unwahrscheinlich, dass die pathologische Anatomie grosse Erfolge zu verzeichnen haben wird.

Der in der Mehrzahl unserer Fälle so rasche and glückliche Verlauf nöthigt uns ans der Beobachtung am Lebenden Schlüsse auf die anatomischen Verhältnisse zn ziehen, und diese Beobachtung hat die grosse Mehrzahl der Beobachter gleich mir zu dem Schluss geführt, dass eine Circulationsstörung, eine Anämie der Hirngefässe die causa proxima sei. Viel weitergehende Schlüsse hat Rosenthal aus der Untersuchung seines Kranken (Nr. 24) gezogen, dessen Motilität und Sensibilität er mittelst Electricität prüfte. „Hierbei stellte sich die seltsame Erscheinung heraus, dass weder die Muskulatur der unteren noch die der oberen Extremitäten auf den lokalen Reiz von angebrachten intensiven Inductionsströmen reagirte; auch bei Reizung der betreffenden Nervenstämme war keine Muskelcontraction noch irgend ein Gefühl von Seiten des Patienten zu erzielen. Aktive Bewegnngsversuche vermochte Patient vorzunehmen; jedoch wurde er nach kurzem Gehen müde. Es stellt sich also - während doch sonst bei rheumatischen (peripherischen) Lähmungen die Muskeln gegen Electricität normal reagiren, in diesem Falle eine fast erloschene electromusculäre Motilität und Sensibilität heraus, und Rosenthal schliesst im vorliegenden Fall auf eine centrale Ursache und zwar auf Druck oder Zerrung der Nervenstämme im Pons oder in der Medulla oblongata, bedingt durch ein seröses Transsudat oder-Oedem. In dem Masse als dieses zur Resorption gelangte, verloren sich die schweren Symptome. Als Patient später geistig und körperlich erstarkte, zeigte es sich auch bei den vorgenommenen späteren Untersuchungen, dass in gleichem Grad auch die periphere Erregbarkeit, als anch die centripetale Leistungsfähigkeit sich wieder restaurirte." $* * *)$

Ich möchte fast bezweifeln, dass der treffliche Wiener Nenropathologe damals schon viele Geisteskranke untersucht und die Eigenschaft der an Melancholie Leidenden gekannt hat, selbst bei intensiven

*) L. j. c. p. 30 .

**) p. 35,39 .

***) Ich citire, da mir das Original nicht zu Gebote steht, nach Fla mm Diss. inaug. p. 43,44 . 
Schmerzen nicht zu zucken.*) Jedenfalls wären weitere Untersuchungen nach dieser Richtung sehr wünschenswerth. Wenn aber selbst seröse Ergüsse da wären, so wäre dies nichts für RheumatismusPsychosen Charakteristisches, da die ganz gleiche Veränderung von Chéron**) bei den Geistesstörungen nach Typhus angenommen wird.

Wir sehen also, dass weder die Form der Rheumatismus-Psychosen noch ihr Verlanf, weder ihre Complicationen noch ihre Prognose oder anatomischer Befund uns nöthigen, diese Geistesstörungen von denen, die nach anderen akuten Krankheiten entstehen, abzutrennen und wie vor 7 Jahren muss ich jetzt, nach Prüfung eines fast verdoppelten Materials erklären: es giebt keine protrahirte rheumatische Hirnaffektion, sondern eine Geistesstörung, die während und nach Rheumatismus acutus wie nach Typhos und Pocken sich entwickelt.

Am Schlusse unserer Arbeit angelangt, wollen wir noch einen Blick auf die Stellung der Geistesstörung innerhalb des vielgestaltigen Gebietes der „nervösen Zufälle im akuten Gelenkrheumatismus" werfen. Wir sehen sie zuweilen aus den akuten Nervenstörungen auf der Höhe der Krankheit hervorgehen, ${ }^{* * *}$ ) nicht selten gleich den akuten nervösen Zufällen der Reconvalescenz $\dagger^{\dagger}$ plötzlich nach der Abfieberung einsetzen; sie bildet sich oft unmerklich aus den Delirien der fieberhaften Erkrankung, zuweilen erst in der scheinbaren Reconvalescenz herans. Zumeist, aber nicht stets, mit Störungen im Gebiet des Respirations- und vor Allem des Circulations-Apparates verbunden - ist die Psychose in ihrer Entwickelung unabhängig von allen diesen Complicationen des Rheumatismus und reiht sich ihnen als gleichwerthiges Glied an.

Indem die Verlanfszeit der Psychose alle Uebergangsformen bietet von dem nur wenige Tage auf der Höhe der Krankheit zu beobachtenden Anfall bis zu der viele Monate die Gelenkaffektion überdanernden Geistesstörung, bildet sie die Vermittelung zwischen den scheinbar so verschiedenen akuten und chronischen Nervenzufällen des Rheumatismus, den nervösen Symptomen auf der Höhe der Krankheit und denen in der

*) Ich glaube, dass ich ein ähnliches Bedenken betreffs der Rosenthal'schen Beobachtung schon anderswo gelesen.

* O) Observations et recherches sur la folie consécutive aux maladies aigues. Paris 1867. p. 50.

***) Oppolzer'sche Beobachtung, erwähnt bei Leidesdorf, Lehrbuch der psychischen Krankheiten. 1867. p. 141.

†) Cfr. üker diese wenig studirte Gruppe Charité-Annalen XIII. p. 105-112. 
Reconvalescenz und führt uns dadurch wie durch die Erkenntniss der sie veranlassenden Ursache zu dem Schlusse, dass auch die räthselhaften akuten nervösen Symptome nicht auf specifisch-rheumatischer Basis ruhen, sondern aus Circulations- und Innervations-Veränderungen des Central-Nervensystems erklärt werden müssen.

Hamburg, December 1873.

\section{Nachtrag.}

\section{Nr. 64. XXXYV. Beobachtung.}

Rheumatismus acutus. Pericarditis Pleuritis duplex. Nach der Abfieberung und dem Aufhören der Gelenkschmerzen heitere Verwirrtheit, 5 Tage dauernd, dann eine Woche noch gegen Abend auftretend. Heilung.

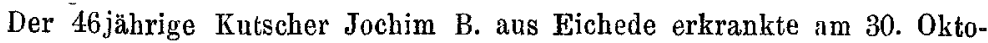
ber 1873 mit Schmerzen in den Knieen, zu denen sich am 2. November solche in den Hand- und Schulter-Gelenken gesellten. Dabei gestörtes Allgemeinbefinden, Appetit- und Schlaflosigkeit, Schweisse.

Am 4. November kam er auf die 4, medicinische Abtheilung des allgemeinen Krankenhauses (Dr. Engel-Reimers).*) Er fieberte mässig $\left(38,4^{0}\right)$, erwies sich als ein kräftiger, gut genährter Mann, dessen beide, sehr empfindlichen Handgelenke durch einen starken Erguss ausgedehnt waren. Ausserdem die Knie, und das linke Schulter-Gelenk afficirt. Herzdämpfung überlagert, Töne rein.

5. November. 37,4-38. Schlaf mässig. Hüftgelenke schmerzhaft.

6. November. 37,6-38,6. Rechte Hand schmerzfrei und abgeschwollen.

7. November. 38,2-38,4. Schlaf ziemlich gat. Linke Hüfte, Hand und Schulter afficirt. In der Gegend der Herzspitze neben den Tönen Reibegeräusch, am Sternalrand dentlich schabend.

8. November. 38,4-38,4. Schlaf gut. Reiben scbwächer.

9. November. 38,2-38,8. Zunge trocken, Abends Schmerzen im rechten Handgelenk. Puls 80-84.

10. November. 38,0-38,6. Umherziehende Schmerzen, Zunge feucht; ge. ringes Reiben am linken Sternalrand.

11. November. 38,2-38,6. Sehr lautes Reiben. Puls 80-96. Zunge trocken.

*) Dessen freundlicher Mittheilung ich die Krankengeschichte verdanke, die ich stellenweise abgekürzt habe. 
12. November. 38,2-39,2. Puls 104-88. Reiben laut. Schmerzen im oberen Theil der Brustwirbelsäule.

In den nächsten Tagen schwankte die Temperatur zwischen 37,8. und 38,8 , der Puls von 82-96, das Reiben blieb hörbar; am 15. December wurde auch ein bis zum angulus scapulae beiderseits reichender pleuritischer Erguss nachgewiesen.

In der folgenden Woche hielt die subfebrile Temperatur (Morgens 37,838,2, Abends $38,0-38,6$ ) an, das Reibungsgeräusch in der Herzgegend blieb, auch liess sich jetzt pericardiales Exsudat durch die Percussion nachweisen. Beim Inspirium nahm das Reibungsgeräusch an Intensität zu, beim Exspirium ab. Ende der Woche war das Allgemeinbefinden gut, die Gelenke schmerzfrei.

Am 24. und 26. November erreichten die Abend-Temperaturen 39,0 resp. 39,2. Der Kranke war abgemagert, die Augen lagen tief, er war traurig gestimmt; der Puls war frequent, stieg am 26. November bis auf 120 .

Am 27. November war die Temperatur $37-38,4^{\circ}$, am 28. November 37,239 (3 Uhr Nachmittags, um $7 \mathrm{Uhr}$ wieder $38,4^{\circ}$ ), stieg am 29 . November nicht über $38^{\circ}$, erreichte am 2. December noch einmal $38,4^{\circ}$ and blieb seitdem vollkommen normal. Während aber jetzt auch die bisher objektiv nachweisbaren Veränderungen (pericardiales und pleuritische Exsudate) sich gebessert batten oder (Gelenkaffektionen) ganz geschwunden waren, fing der Kranke am Morgen des 4. December an irre zu reden, behauptete, dass er zu Hause gewesen sei, er müsse sogar nochmals zurück, habe dort etwas vergessen. Er sei nicht im Krankenhause, das Zimmer knomme ihm verändert vor. Mit einem Wort: der Patient ist voll Wahnideen and nimmt es sehr übel, wenn man diese anzweifelt.

Am 6. December gab er selbst auf ganz einfache Fragen verkehrte Antworten, spricht Nachts im Schlafe, Der Zustand von Verwirrtheit neben entschiedener Euphorie hielt bis zum 8. December an. Von diesem Tage an erschien das Sensorium Tags über frei, Abends zeigten sich aber noch immer Delirien, obschon die Temperatur nur zwischen $36,4^{0}$ und $37,4^{0}$ schwankte.

Vom 16. December an blieb das Sensorium frei, nur war die Gemüthsstimmung vielleicht etwas abnorm heiter. Das pericardiale wie das pleuritische Exsudat waren resorbirt und der Kranke konnte am 21. December geheilt entlassen werden.

Der Fall ist ein exquisites Beispiel von Inanitions-Delirien, scheinbar nur wenige Tage (4. bis 8 . December) bestehend, doch muss man die Periode gestörter geistiger Thätigkeit wohl bis zum 16. December, also fast 2 Wochen berechnen.

Nur wenige Fülle zeigen so klal wie dieser, dass die Gelenkaffektion, der rheumatische Process als solcher, mit der Geistesstörang nichts zu thun hat; beinah 2 Wochen hindurch war der Kranke frei von Gelenkaffektion, ehe die Verwirrtheit sich zeigte; aber er war heruntergekommen durch das lange Krankenlager, das Fieber, die Brust- 
Die Geistesstörungen im Verlaufe des akuten Gelenkrheumatismus. 697 and Herzaffektionen und in einem Znstand klar ausgesprochener Inanition.

Auch die Form der Geistesstörung ist insofern ron besonderem Interesse, als sie sich der Verwirrtheit mit einzelnen scharf ausgesprochenen Wahnideen anreiht, wie sie beim Typhus häufiger ist, während die Rheumatismus-Litteratur daran arm ist. 